\title{
AN ANALYSIS OF DELAY-DEPENDENT STABILITY FOR ORDINARY AND PARTIAL DIFFERENTIAL EQUATIONS WITH FIXED AND DISTRIBUTED DELAYS*
}

\author{
CHENGMING HUANG ${ }^{\dagger}$ AND STEFAN VANDEWALLE ${ }^{\dagger}$
}

\begin{abstract}
This paper is concerned with the study of the stability of ordinary and partial differential equations with both fixed and distributed delays, and with the study of the stability of discretizations of such differential equations. We start with a delay-dependent asymptotic stability analysis of scalar ordinary differential equations with real coefficients. We study the exact stability region of the continuous problem as a function of the parameters of the model. Next, it is proved that a time discretization based on the trapezium rule can preserve the asymptotic stability for the considered set of test problems. In the second part of the paper, we study delay partial differential equations. The stability region of the fully continuous problem is analyzed first. Then a semidiscretization in space is applied. It is shown that the spatial discretization leads to a reduction of the stability region when the standard second-order central difference operator is employed to approximate the diffusion operator. Finally we consider the delay-dependent stability of the fully discrete problem, where the partial differential equation is discretized both in space and in time. Some numerical examples and further discussions are given.
\end{abstract}

Key words. delay integrodifferential equations, delay partial differential equations, stability region, trapezium rule

AMS subject classifications. 65L20, 65M10, 65M20

DOI. $10.1137 / \mathrm{S} 1064827502409717$

1. Introduction. The stability of discretization methods plays an important role in the numerical solution of delay differential equations. In the last twenty years, many papers have dealt with this topic and a significant number of important results have been found. For a description of state-of-the-art, we refer the reader to the papers by Baker $[2,3]$, to the book by Bellen and Zennaro [6], and to the extensive bibliography therein.

One of the interesting problems in stability analysis is the investigation of the delay-dependent stability region of numerical methods. For ordinary differential equations (ODEs) with a fixed delay, this subject was first discussed by Cryer [8], Al Mutib [1], van der Houwen and Sommeijer [18], and Calvo and Grande [7]. More recently, a series of results was obtained by Guglielmi [10, 11, 12], Guglielmi and Hairer [13, 14], and Maset [17]. For ODEs with a distributed delay, a numerical investigation was given by Baker and Ford [4]. For ODEs with both fixed and distributed delays, Koto [16] recently investigated the delay-independent stability of Runge-Kutta methods. We have not found any result on delay-dependent stability of such equations. The numerical solution of partial differential equations (PDEs) with delays has only recently attracted the attention of several authors. Zubik-Kowal and Vandewalle [21] have studied a special class of iterative methods, i.e., waveform relaxation methods, for solving semidiscrete delay PDEs. Their paper is not concerned with stability issues of the discretization, but rather with the convergence of the iterative ODE solution method. Zubik-Kowal [20] discussed the contractivity of $\theta$-methods under the as-

\footnotetext{
* Received by the editors June 18, 2002; accepted for publication (in revised form) August 29, 2003; published electronically March 3, 2004.

http://www.siam.org/journals/sisc/25-5/40971.html

${ }^{\dagger}$ Katholieke Universiteit Leuven, Department of Computer Science, Celestijnenlaan 200A, B3001 Leuven, Belgium (Chengming.Huang@cs.kuleuven.ac.be, Stefan.Vandewalle@cs.kuleuven.ac.be).
} 
sumption that the semidiscrete system with a fixed delay is asymptotically stable for every positive delay.

In the first part of this paper, we discuss the numerical stability of real coefficient scalar ODEs with both fixed and distributed delays. On the basis of the investigation of the analytical stability region, the stability region of the trapezium rule is derived. It is proved that the trapezium rule preserves many features of the analytical stability region, including asymptotic stability. In the second part of this paper, we consider the numerical delay-dependent stability of PDEs of parabolic type, with both fixed and distributed delays. We derive the analytical stability region of the PDE and also investigate the stability of the semidiscrete and fully discrete problems. To this end we employ standard second-order central differences to discretize the spatial derivative and the trapezium rule to discretize the time derivative. Our result shows that the spatial discretization leads to a reduction of the size of the stability region. This is different from the case of PDEs without delays. Hence, also the corresponding full discretization based on the use of the trapezium rule cannot completely preserve the asymptotic stability of the original PDE.

This paper is organized as follows. In section 2, we study the scalar ODE case for problems with fixed and distributed delays. In section 3, we investigate the stability of a class of linear PDE problems and the stability of the semidiscrete and fully discrete cases. In section 4, some numerical examples and further discussions are given. Finally, we draw some conclusions in section 5 .

2. Stability of ordinary delay differential equations. This section consists of two parts. First, we study the analytical stability of a model equation. Then we investigate the discretized stability based on the trapezium rule.

2.1. Stability region of the continuous problem. In order to obtain the exact boundary of stability region, we will study many properties of the characteristic equation, including the location of the boundary locus and the number of roots in the right half-plane.

2.1.1. Study of the imaginary roots of the characteristic equation. We study the asymptotic stability of the solution to the equation

$$
y^{\prime}(t)=\alpha y(t)+\beta y(t-\tau)+\gamma \int_{-\tau}^{0} y(t+s) d s, \quad t>0,
$$

where $\alpha, \beta, \gamma \in \mathbb{R}, \tau \in \mathbb{R}^{+}$, and $y(t)=g(t)$ on $[-\tau, 0]$. The results obtained for this very general equation will be specialized in section 2.1.3 towards the cases of fixed delay problems $(\gamma=0)$, distributed delay problems $(\beta=0)$, and pure delay problems $(\alpha=0)$. By looking at solutions of the form $y(t)=\exp (\lambda t)$ we are led to the characteristic equation

$$
\lambda=\alpha+\beta \exp (-\tau \lambda)+\gamma \int_{-\tau}^{0} \exp (\lambda s) d s .
$$

The asymptotic stability of the zero solution to (2.1) is equivalent to the condition that all the roots of algebraic equation (2.2) have negative real parts. Below, we analyze these roots, following the ideas in the work by Diekmann et al. [9, Chap. XI]. Writing $\lambda=\mu+i \nu$ with $\mu, \nu \in \mathbb{R}$, we find two real equations,

$$
\mu=\alpha+\beta \exp (-\tau \mu) \cos (\tau \nu)+\gamma \int_{-\tau}^{0} \exp (\mu s) \cos (\nu s) d s
$$




$$
\nu=-\beta \exp (-\tau \mu) \sin (\tau \nu)+\gamma \int_{-\tau}^{0} \exp (\mu s) \sin (\nu s) d s,
$$

for the real and imaginary parts of the eigenvalue $\lambda$. Now we concentrate on roots which lie on the imaginary axis, the so-called critical roots, i.e., with $\mu=0$. With also $\nu=0$, one finds that there exists a root $\lambda=0$ if the parameters $\alpha, \beta$, and $\gamma$ satisfy

$$
\alpha+\beta+\gamma \tau=0 .
$$

For a given value of $\beta$ this set can be interpreted as a line in the $(\alpha, \gamma)$-plane. This line will be denoted by $C_{*}(\beta)$. Setting $\theta=\tau \nu$, we find that the following parameter sets $(\alpha(\theta), \beta, \gamma(\theta))$ with $\theta \neq \pm 2 k \pi, k=1,2, \ldots$, lead to a purely imaginary root $\lambda$ :

$$
\alpha(\theta)=\beta+\frac{\theta \sin \theta}{\tau(1-\cos \theta)} \quad \text { and } \quad \gamma(\theta)=\frac{-\theta(\theta+\beta \tau \sin \theta)}{\tau^{2}(1-\cos \theta)} .
$$

Since these functions have singularities for $\theta= \pm 2 k \pi$, we introduce the intervals

$$
J_{k}=(2 k \pi, 2(k+1) \pi)
$$

and denote by $C_{k}(\beta)$ the curve in the $(\alpha, \gamma)$-plane for a fixed $\beta$-value, parameterized by $\theta$ for $\theta \in J_{k}$. We will adhere to the convention that $C_{0}(\beta)$ also contains the limit point for $\theta \rightarrow 0$ :

$$
(\alpha(0), \gamma(0))=\left(\beta+\frac{2}{\tau}, \frac{-2(1+\beta \tau)}{\tau^{2}}\right) .
$$

The functions in (2.6) are even functions in $\theta$, which reflects the fact that the complex roots appear in complex conjugate pairs. Hence, we may restrict further attention to $k \geq 0$, i.e., $\nu \geq 0$. As an illustration, the curves $C_{k}(\beta)$ are shown in Figure 1 for two different values of $\beta$ and for $\tau=1$.

We will now analyze some important properties of these curves, in a series of four (rather technical) lemmas. These will show that the configuration and features of the curves in Figure 1 are typical. We show that the curves $C_{k}(\beta)$ do not intersect, and that they can be ordered according to $k$. We determine their limiting values and consider the location of curve $C_{*}(\beta)$ versus the $C_{0}(\beta)$-curve.

Lemma 2.1. The curves $C_{k}(\beta)$ do not intersect.

Proof. Suppose that there exist $\theta_{1} \in J_{k}$ and $\theta_{2} \in J_{l}$ with $k \neq l$ such that $\alpha\left(\theta_{1}\right)=\alpha\left(\theta_{2}\right)$ and $\gamma\left(\theta_{1}\right)=\gamma\left(\theta_{2}\right)$, or

$$
\frac{\theta_{1} \sin \theta_{1}}{1-\cos \theta_{1}}=\frac{\theta_{2} \sin \theta_{2}}{1-\cos \theta_{2}} \quad \text { and } \quad \frac{\theta_{1}^{2}+\beta \tau \theta_{1} \sin \theta_{1}}{1-\cos \theta_{1}}=\frac{\theta_{2}^{2}+\beta \tau \theta_{2} \sin \theta_{2}}{1-\cos \theta_{2}} .
$$

Substituting the first equality into the second one yields

$$
\frac{1-\cos \theta_{1}}{\theta_{1}^{2}}=\frac{1-\cos \theta_{2}}{\theta_{2}^{2}}
$$

Multiplying both sides by the corresponding sides of the left equality in (2.9) and squaring, one arrives at

$$
\frac{\sin ^{2} \theta_{1}}{\theta_{1}^{2}}=\frac{\sin ^{2} \theta_{2}}{\theta_{2}^{2}}
$$



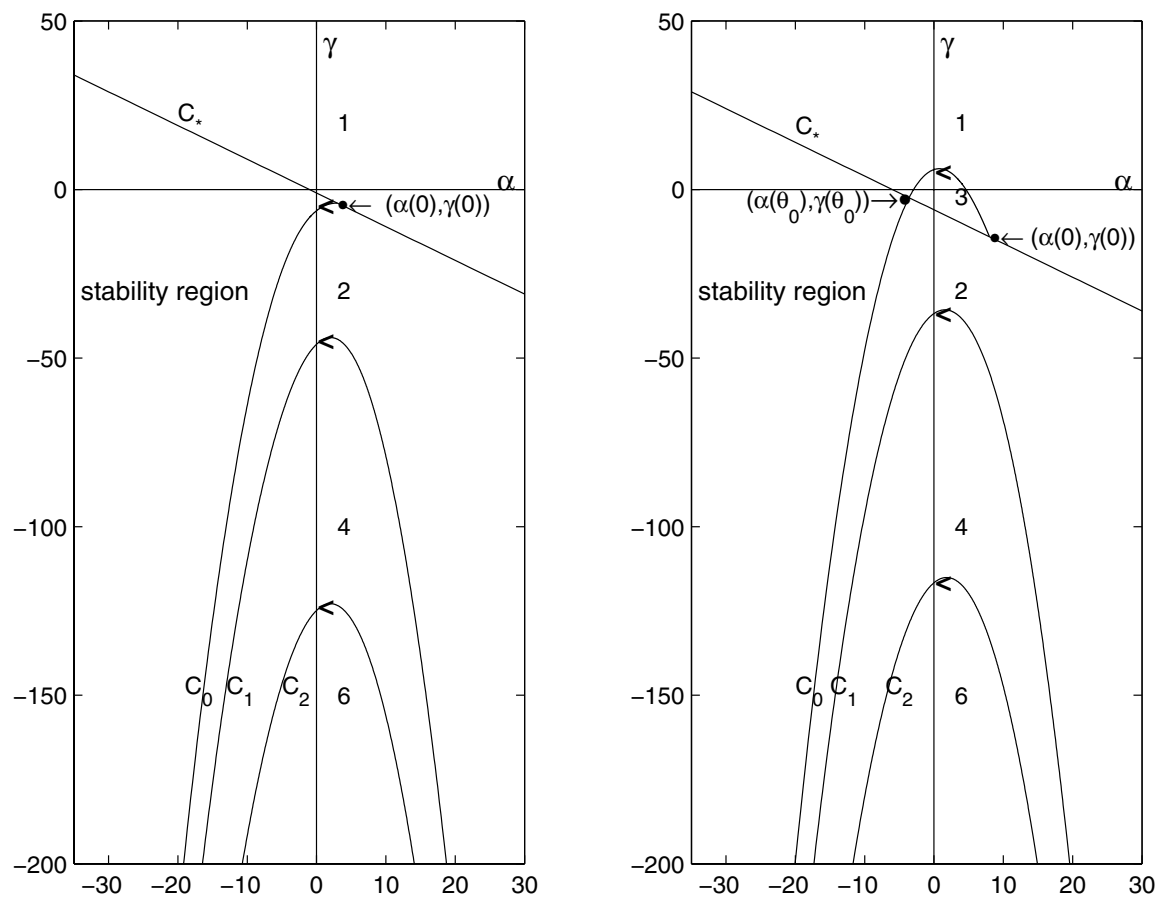

FIG. 1. The curves $C_{k}(\beta)$ in the $(\alpha, \gamma)$-plane for $\tau=1$ (left: $\beta=1$; right: $\beta=6$ ). The arrows along the curves refer to the direction of increasing $\theta$. The numbers in the different regions bordered by the curves indicate the number of roots in the right half-plane.

Subtracting (2.11) from (2.10) yields

$$
\frac{\cos \theta_{1}\left(\cos \theta_{1}-1\right)}{\theta_{1}^{2}}=\frac{\cos \theta_{2}\left(\cos \theta_{2}-1\right)}{\theta_{2}^{2}} .
$$

Equations (2.10) and (2.12) lead to $\cos \theta_{1}=\cos \theta_{2}$. Hence, (2.10) can only be satisfied when $\theta_{1}=\theta_{2}$, which contradicts our earlier assumption.

Lemma 2.2. The curve $C_{k}(\beta)$ intersects the line $\alpha=\beta$ exactly once. The $\gamma$ coordinate $\gamma_{k}$ of the intersection satisfies $\gamma_{k+1}<\gamma_{k}$.

Proof. When $\theta \in J_{k}$, the equality $\alpha(\theta)=\beta$ implies $\theta=(2 k+1) \pi$. Hence,

$$
\gamma_{k}=\frac{-(2 k+1)^{2} \pi^{2}}{2 \tau^{2}}
$$

which satisfies $\gamma_{k+1}<\gamma_{k}$. This completes the proof.

LEMMA 2.3.

$$
\lim _{\theta \rightarrow 2 k \pi+0} \alpha(\theta)=+\infty, \quad \lim _{\theta \rightarrow 2 k \pi-0} \alpha(\theta)=-\infty, \quad \lim _{\theta \rightarrow 2 k \pi \pm 0} \gamma(\theta)=-\infty, \quad k=1,2, \ldots
$$

Lemma 2.4. The curve $C_{0}(\beta)$ intersects $C_{*}(\beta)$ at $(\alpha(0), \gamma(0))$. If $\beta \tau>2$, it also intersects at another point, $\left(\alpha\left(\theta_{0}\right), \gamma\left(\theta_{0}\right)\right)$, with $\theta_{0}$ as defined in (2.13).

Proof. For any given $\beta \tau>2$, there exists a unique $\theta_{0} \in(0,2 \pi)$ such that

$$
\beta \tau=\frac{\theta_{0}\left(\theta_{0}-\sin \theta_{0}\right)}{2-2 \cos \theta_{0}-\theta_{0} \sin \theta_{0}} .
$$


A straightforward computation shows that the corresponding point $\left(\alpha\left(\theta_{0}\right), \gamma\left(\theta_{0}\right)\right)$ lies on the line $C_{*}(\beta)$.

2.1.2. Number of roots in the right half-plane. Based on (2.3) and (2.4) one easily verifies the following result, which guarantees that the roots with positive real parts are located in a compact region, with size depending on the problem parameters.

Lemma 2.5. Let $\lambda$ be a root of the characteristic equation (2.2) with $\operatorname{Re} \lambda>0$. Then

$$
\operatorname{Re} \lambda<\alpha+|\beta|+|\gamma \tau| \text { and } \operatorname{Im} \lambda<|\beta|+|\gamma \tau| .
$$

From the continuity of the roots as a function of the coefficients of the characteristic equation (see, e.g., [9]), we may conclude that the number of roots in the right half-plane is constant in the regions of the $(\alpha, \gamma)$-plane bounded by $C_{k}(\beta)$ and the line $C_{*}(\beta)$. It is easy to verify that all roots of $(2.2)$ are in the left half-plane when $\alpha=-|\beta|-1, \gamma=0$. Now we study the crossing of a pair of imaginary roots into the right half-plane and determine the direction of the corresponding trajectory in the $(\alpha, \gamma)$-plane. To this end, we define two functions based on (2.3) and (2.4):

$$
\begin{aligned}
& G_{1}(\alpha, \gamma, \mu, \nu)=-\mu+\alpha+\beta \exp (-\tau \mu) \cos (\tau \nu)+\gamma \int_{-\tau}^{0} \exp (\mu s) \cos (\nu s) d s \\
& G_{2}(\alpha, \gamma, \mu, \nu)=-\nu-\beta \exp (-\tau \mu) \sin (\tau \nu)+\gamma \int_{-\tau}^{0} \exp (\mu s) \sin (\nu s) d s .
\end{aligned}
$$

Calculating the Jacobian matrix $M$ defined by

$$
M=\left[\begin{array}{ll}
\frac{\partial G_{1}}{\partial \alpha} & \frac{\partial G_{1}}{\partial \gamma} \\
\frac{\partial G_{2}}{\partial \alpha} & \frac{\partial G_{2}}{\partial \gamma}
\end{array}\right]_{\mu=0}
$$

and computing its determinant, one finds

$$
\operatorname{det} M=\frac{\cos \tau \nu-1}{\nu}<0 .
$$

Now a result from [9, Chap. XI, Prop. 2.13] can be applied.

LEMma 2.6. The critical roots are in the right half-plane in the $(\alpha, \gamma)$ parameter region to the left of the critical curve, when we follow this curve in the direction of increasing $\theta$ when $\operatorname{det} M<0$ and to the right when $\operatorname{det} M>0$.

Hence, the critical roots move into the right half-plane when moving away in the parameter space to the left of $C_{k}(\beta)$, with "left" as determined with respect to a counterclockwise tracking of $C_{k}(\beta)$. In Figure 1, the number of roots in the right half-plane is indicated for each separate parameter region. As a consequence of the above analysis, we are able to state the main result of this subsection.

THEOREM 2.7. The zero solution of (2.1) is asymptotically stable, i.e., all the roots of (2.2) have negative real parts iff

(a) $\alpha<\beta+\frac{2}{\tau}$ and

(b) $\tau(\alpha+\beta)<-\gamma \tau^{2}<\frac{\theta(\theta+\beta \tau \sin \theta)}{1-\cos \theta}$, where $\theta$ is the root of $\alpha=\beta+\frac{\theta \sin \theta}{\tau(1-\cos \theta)}$ such that $\theta \in(0,2 \pi)$.

Proof. The stability region lies below $C_{*}(\beta)$ and above $C_{0}(\beta)$. Therefore, the results immediately follow from (2.5), (2.6), and (2.8). 

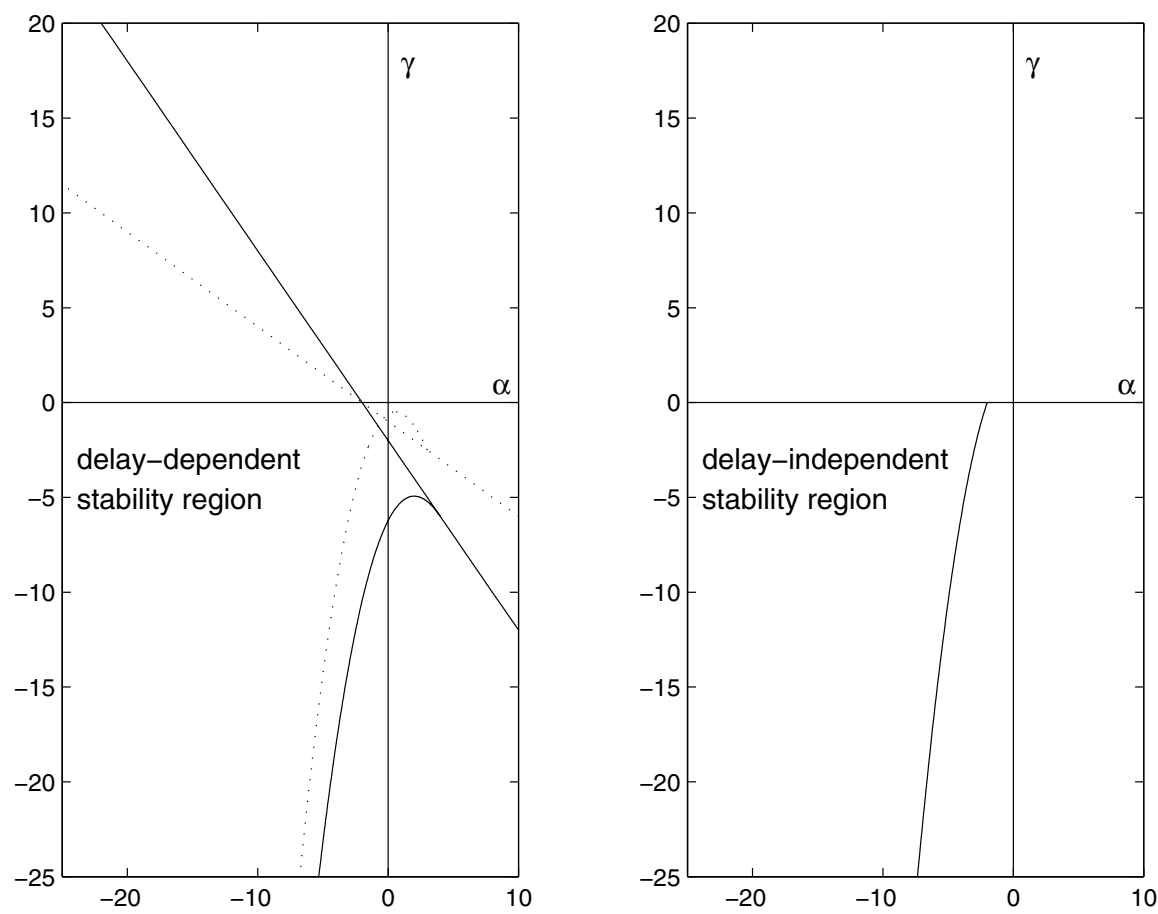

FIG. 2. Delay-dependent stability region for various $\tau$-values (left: $\tau=1$ (solid) and $\tau=2$ (dotted)) and delay-independent stability region (right) for $\beta=2$.

Theorem 2.7 can be seen as a generalization of the delay-independent stability condition derived in [16] by Koto for problem (2.1). We consider the case $\gamma \neq 0$. If condition (a) and the left inequality of condition (b) are to hold for every $\tau>0$, we must have that $\alpha \leq \beta$ and $\gamma<0$. Squaring the equality $\alpha=\beta+\frac{\theta \sin \theta}{\tau(1-\cos \theta)}$ and substituting into the right inequality of condition (b) lead to an equivalent inequality,

$$
-\gamma<\frac{\alpha^{2}-\beta^{2}}{2}+\frac{\theta^{2}}{2 \tau^{2}}
$$

If this is to hold independent of $\tau$, then we have that $-\gamma \leq \frac{\alpha^{2}-\beta^{2}}{2}$, which further gives $\alpha<-|\beta| \leq 0$. Hence, we have derived a result, originally due to Koto, as a corollary of our stability theorem.

Corollary 2.8. Consider the case $\gamma \neq 0$. The zero solution of (2.1) is asymptotically stable independently of the delay $\tau$ iff

$$
\alpha<0, \quad \gamma<0, \quad-\gamma \leq \frac{\alpha^{2}-\beta^{2}}{2} .
$$

Because we consider delay-dependent stability, the stability regions we obtain are larger than those in [16]. The difference between the delay-dependent and the delay-independent stability regions for the case $\beta=2$ is illustrated in Figure 2.

Our analysis of the stability of delay PDEs in section 3 will require some more detailed information about the curves $C_{k}(\beta)$. Accurate knowledge of the location of extremal values will be important, as will be, in particular, knowledge about the 
monotonicity. This will be investigated next. It is easily verified that $\alpha(\theta)$ is strictly decreasing with respect to $\theta$ in every $J_{k}, k=0,1,2, \ldots$ The $\gamma(\theta)$-function, on the other hand, satisfies

$$
\gamma^{\prime}(\theta)=\frac{-\theta(2-2 \cos \theta-\theta \sin \theta)+\beta \tau(1-\cos \theta)(\theta-\sin \theta)}{\tau^{2}(1-\cos \theta)^{2}},
$$

which shows that $\gamma(\theta)$ has exactly one extremum with respect to $\theta$ along the curves $C_{k}(\beta)$ when $k>0$. The more intricate and important case $\theta \in J_{0}$ is now considered separately. When $\beta \tau \leq 1$, the function $\gamma(\theta)$ is strictly decreasing. When $\beta \tau>1$, there exists a unique $\bar{\theta}_{0} \in(0,2 \pi)$ such that $\gamma^{\prime}\left(\bar{\theta}_{0}\right)=0$, namely, the root of

$$
\beta \tau=\frac{\bar{\theta}_{0}\left(2-2 \cos \bar{\theta}_{0}-\bar{\theta}_{0} \sin \bar{\theta}_{0}\right)}{\left(1-\cos \bar{\theta}_{0}\right)\left(\bar{\theta}_{0}-\sin \bar{\theta}_{0}\right)} .
$$

Hence, $\gamma(\theta)$ has one extremum $\gamma\left(\bar{\theta}_{0}\right)$. This value $\bar{\theta}_{0}$ satisfies

$$
\alpha\left(\bar{\theta}_{0}\right)=\frac{\bar{\theta}_{0}\left(2-2 \cos \bar{\theta}_{0}-\sin ^{2} \bar{\theta}_{0}\right)}{\tau\left(1-\cos \bar{\theta}_{0}\right)\left(\bar{\theta}_{0}-\sin \bar{\theta}_{0}\right)}>0 .
$$

Define the constant $\alpha_{0}$ as follows

$$
\alpha_{0}=\left\{\begin{array}{cc}
\alpha(0) & \text { if } \beta \tau \leq 1, \\
\alpha\left(\bar{\theta}_{0}\right) & \text { otherwise } .
\end{array}\right.
$$

Therefore, for any given $\beta \in \mathbb{R}, C_{0}(\beta)$ is strictly monotonic in the $(\alpha, \gamma)$-plane when $\alpha \leq \alpha_{0}$. In particular, $C_{0}(\beta)$ is strictly monotonic in the left half of the $(\alpha, \gamma)$-plane. This leads to the following results, which will be used in section 3 .

THEOREM 2.9. Suppose all roots of (2.2) have negative real parts for given $\alpha$, $\beta, \gamma \in \mathbb{R}$, with $\alpha \leq \alpha_{0}$ for $\alpha_{0}$ as defined in (2.14). Then for any $x \leq \alpha$, all the roots $\lambda$ of the equation

$$
\lambda=x+\beta \exp (-\tau \lambda)+\gamma \int_{-\tau}^{0} \exp (\lambda s) d s
$$

have negative real parts too.

COROLlary 2.10. Suppose all roots of (2.2) have negative real parts for given $\alpha \leq 0, \beta, \gamma \in \mathbb{R}$. Then for any $x \leq \alpha$, all roots of (2.15) have negative real parts too.

2.1.3. Some special cases. Next, we consider some special cases and show that our framework enables us to rederive some specialized results from the delay equation literature. First we consider a differential equation with a distributed delay,

$$
\begin{cases}y^{\prime}(t)=\alpha y(t)+\gamma \int_{-\tau}^{0} y(t+s) d s, & t>0, \\ y(t)=g(t), & t \in[-\tau, 0] .\end{cases}
$$

This is the basic equation studied by Baker and Ford in [4]. A direct application of Theorem 2.7 for $\beta=0$ allows us to regain a result given in the above reference.

COROLlary 2.11. The zero solution of (2.16) is asymptotically stable iff

(a) $\alpha \tau<2$ and

(b) $\tau \alpha<-\gamma \tau^{2}<\frac{\theta^{2}}{1-\cos \theta}$, 

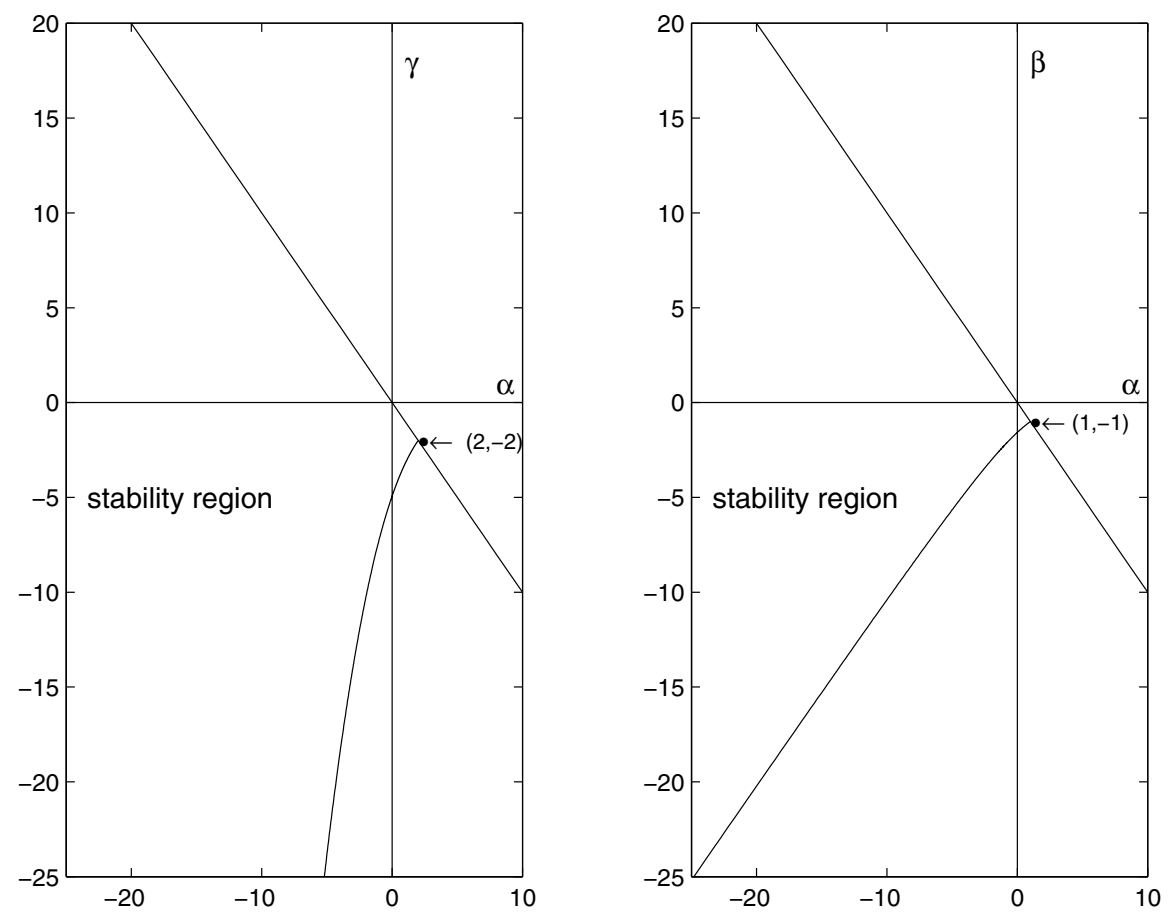

FIG. 3. Stability region of (2.16) (left) and stability region of (2.17) (right) for $\tau=1$.

where $\theta$ is the root of $\alpha=\frac{\theta \sin \theta}{\tau(1-\cos \theta)}$ such that $\theta \in(0,2 \pi)$.

The stability region of (2.16) for $\tau=1$ is shown in the left panel of Figure 3. Next, we study the case of an equation with a fixed delay, i.e., with $\gamma=0$,

$$
\begin{cases}y^{\prime}(t)=\alpha y(t)+\beta y(t-\tau), & t>0, \\ y(t)=g(t), & t \in[-\tau, 0] .\end{cases}
$$

This is a classical model equation in the delay equation literature. In this case, condition (b) in Theorem 2.7 simplifies to

$$
\alpha+\beta<0 \text { and } \theta+\beta \tau \sin \theta>0,
$$

where $\theta$ is the root of

$$
\alpha=\beta+\frac{\theta \sin \theta}{\tau(1-\cos \theta)}
$$

such that $\theta \in(0,2 \pi)$. If $\alpha \leq \beta$, i.e., $\theta \in[\pi, 2 \pi)$, from the left inequality in (2.18) and (2.19) it follows that

$$
2 \beta+\frac{\theta \sin \theta}{\tau(1-\cos \theta)}<0
$$

which guarantees that the right inequality of (2.18) holds. Therefore, we have the following well-known result (see, e.g., Diekmann et al. [9]). 


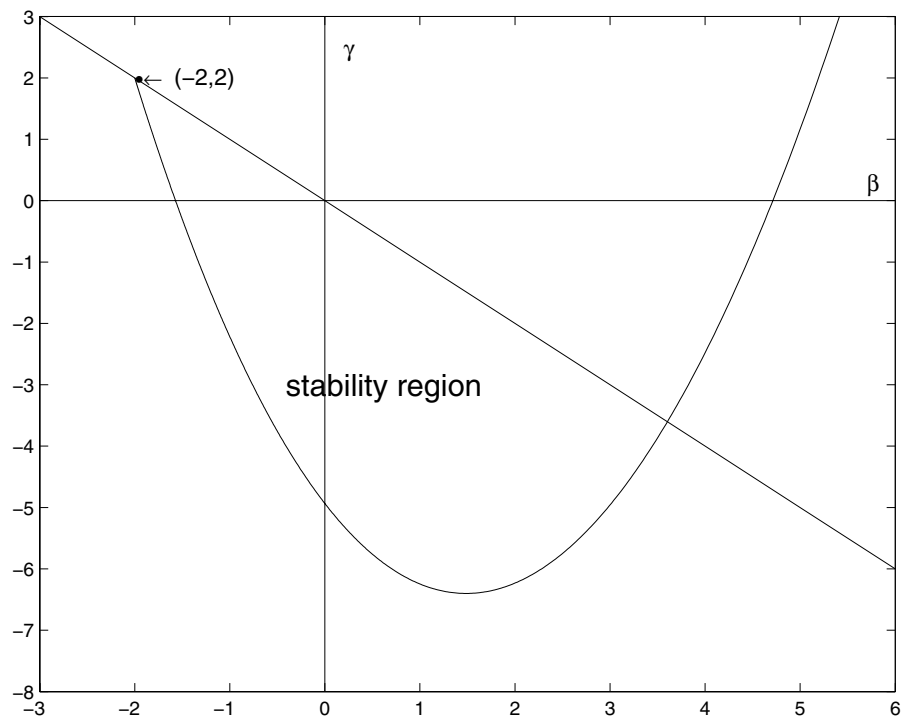

FIG. 4. Stability region of the analytical solution of (2.20) for $\tau=1$.

COROLlary 2.12. The zero solution of (2.17) is asymptotically stable iff

(a) $\alpha \tau<1$ and

(b) $\tau \alpha<-\tau \beta<\frac{\theta}{\sin \theta}$,

where $\theta$ is the root of $\theta \cos \theta=\tau \alpha \sin \theta$ such that $\theta \in(0, \pi)$.

The stability region of (2.17) for $\tau=1$ is shown in the right panel of Figure 3. Finally, the case of a pure delay equation is considered; i.e., with $\alpha=0$,

$$
\begin{cases}y^{\prime}(t)=\beta y(t-\tau)+\gamma \int_{-\tau}^{0} y(t+s) d s, & t>0, \\ y(t)=g(t), & t \in[-\tau, 0] .\end{cases}
$$

For the case $\gamma=0$, a numerical stability analysis of this equation was first given by Cryer [8] and by van der Houwen and Sommeijer [18]. Equation (2.20) has a finite region of asymptotic stability. An application of Theorem 2.7 leads to the following corollary.

COROLlaRY 2.13. The zero solution of (2.20) is asymptotically stable iff

(a) $\beta \tau+2>0$ and

(b) $\beta \tau<-\gamma \tau^{2}<\frac{-\theta^{2} \cos \theta}{1-\cos \theta}$, where $\theta$ is the root of $\beta=\frac{-\theta \sin \theta}{\tau(1-\cos \theta)}$ such that $\theta \in(0,2 \pi)$.

The stability region of (2.20) for $\tau=1$ is shown in Figure 4.

2.2. Stability region of the trapezium rule discretization. In this subsection, we first give the stability region of the trapezium rule. Then we prove that the analytical stability region is a subset of the numerical stability region.

2.2.1. Study of the unit modulus roots of the characteristic equation. We apply the trapezium rule with constant and consistent stepsize $h=\tau / m$ to $(2.1)$, with $m$ a positive integer. We denote by $y_{n-m}$ the approximation to $y\left(t_{n}-\tau\right)$ and 
use the repeated trapezium rule to approximate the integral term. This leads to

$$
\begin{aligned}
y_{n+1}-y_{n}= & \frac{h}{2}\left[\alpha y_{n+1}+\beta y_{n+1-m}+\frac{\gamma h}{2}\left(\sum_{j=0}^{m-1} y_{n+1-j}+\sum_{j=1}^{m} y_{n+1-j}\right)\right] \\
& +\frac{h}{2}\left[\alpha y_{n}+\beta y_{n-m}+\frac{\gamma h}{2}\left(\sum_{j=0}^{m-1} y_{n-j}+\sum_{j=1}^{m} y_{n-j}\right)\right] .
\end{aligned}
$$

The characteristic equation of the above difference equation is given by

$$
1-z^{-1}=\frac{h}{2}\left(1+z^{-1}\right)\left[\alpha+\beta z^{-m}+\frac{\gamma h}{2}\left(1+z^{-1}\right) \sum_{j=0}^{m-1} z^{-j}\right] .
$$

The numerical solution of (2.21) is asymptotically stable for any initial values iff all roots $z$ of the characteristic equation (2.22) satisfy $|z|<1$. Since $z$ in (2.22) depends continuously on $\alpha, \beta$, and $\gamma$, we apply the so-called boundary locus technique (cf. $[4,5])$ to analyze the roots of $(2.22)$. This technique has been successfully used to analyze the numerical delay-dependent stability region for ODEs with a fixed delay (cf. $[10,11,12,13,14,17]$ ) and for ODEs with distributed delay (cf. [4]).

Similar to the case of the continuous system in section 2.1, we fix $\beta$ and consider the stability region in the $(\alpha, \gamma)$-plane. To distinguish this from the continuous case, we will use "barred" notation to denote any quantities or functions related to the discrete case. For example, we use $\bar{\alpha}$ and $\bar{\gamma}$ as parameters for the curves on which the characteristic equation $(2.22)$ has at least one root $z$ on the unit circle. Let $z^{-1}=\exp (i \varphi)$. Because $\alpha, \beta$, and $\gamma$ are real, we can restrict our analysis to $\varphi \in[0, \pi]$. The value $z=1$, i.e., $\varphi=0$, gives the line $\bar{C}_{*}(\beta)$ with equation $\bar{\alpha}+\beta+\bar{\gamma} \tau=0$, which is identical to the line $C_{*}(\beta)$ in the case of the continuous problem. Obviously, $z=-1$, i.e., $\varphi=\pi$, is not a root of (2.22). It is easily verified that, except for $z=1$, any other $z$ satisfying $z^{m}=1$ is not a root of (2.22). For other $\varphi$-values, we have

$\frac{-i \sin \varphi}{1+\cos \varphi}=\frac{h}{2}\left[\bar{\alpha}+\beta(\cos m \varphi+i \sin m \varphi)+\frac{\bar{\gamma} h}{2} \frac{i(1+\cos \varphi)}{\sin \varphi}(1-\cos m \varphi-i \sin m \varphi)\right]$.

Separating real and imaginary parts yields

$$
\begin{aligned}
0 & =\bar{\alpha}+\beta \cos m \varphi+\frac{\bar{\gamma} h}{2} \frac{(1+\cos \varphi) \sin m \varphi}{\sin \varphi}, \\
\frac{-\sin \varphi}{1+\cos \varphi} & =\frac{h}{2}\left[\beta \sin m \varphi+\frac{\bar{\gamma} h}{2} \frac{(1+\cos \varphi)(1-\cos m \varphi)}{\sin \varphi}\right] .
\end{aligned}
$$

Solving for $\bar{\alpha}$ and $\bar{\gamma}$, one obtains

$$
\begin{aligned}
& \bar{\alpha}_{m}(\varphi)=\beta+\frac{2 m \sin \varphi \sin m \varphi}{\tau(1+\cos \varphi)(1-\cos m \varphi)}, \\
& \bar{\gamma}_{m}(\varphi)=\frac{-2 m \sin \varphi(2 m \sin \varphi+\beta \tau \sin m \varphi(1+\cos \varphi))}{\tau^{2}(1+\cos \varphi)^{2}(1-\cos m \varphi)} .
\end{aligned}
$$

Define the intervals

$$
\bar{J}_{k}=\left(\frac{2 k \pi}{m}, \frac{2(k+1) \pi}{m}\right), \quad k=0,1, \ldots,\left\lfloor\frac{m}{2}\right\rfloor-1,
$$




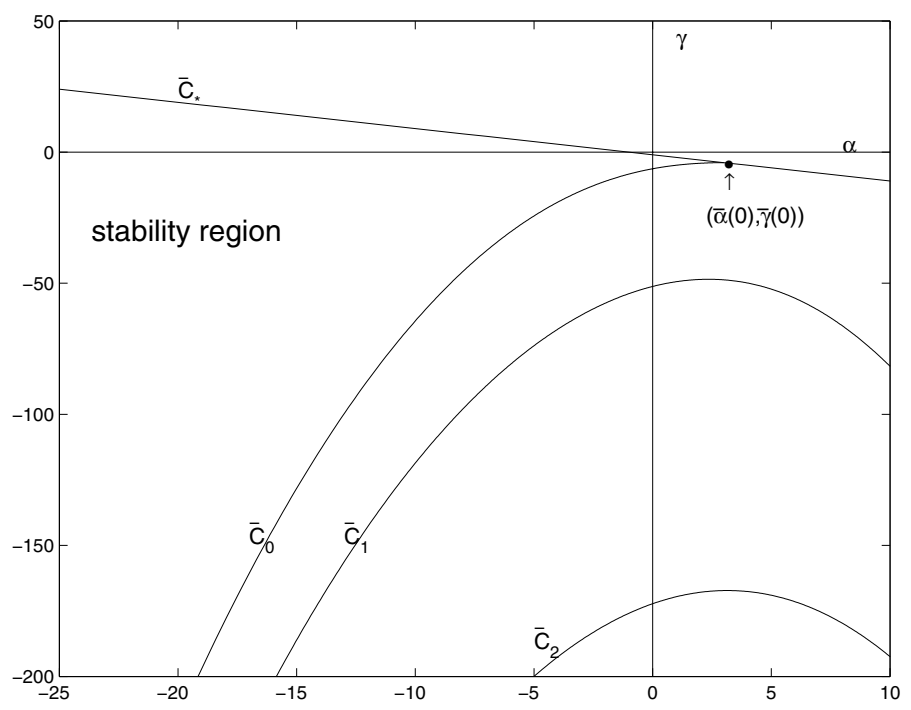

FIG. 5. The curves $\bar{C}_{k}(\beta)$ in the $(\alpha, \gamma)$-plane for $\beta=1, \tau=1$, and $m=12$.

where $\lfloor x\rfloor$ stands for the integer part of $x$. When $m$ is odd, we additionally define

$$
\bar{J}_{\left\lfloor\frac{m}{2}\right\rfloor}=\left(\frac{(m-1) \pi}{m}, \pi\right) .
$$

For every fixed $\beta$, we denote by $\bar{C}_{k}(\beta)$ the curve in the $(\alpha, \gamma)$-plane parameterized by $\varphi$ for $\varphi \in \bar{J}_{k}$, with the convention that $\bar{C}_{0}(\beta)$ also contains the limit point for $\varphi \rightarrow 0$ :

$$
\left(\bar{\alpha}_{m}(0), \bar{\gamma}_{m}(0)\right)=\left(\beta+\frac{2}{\tau}, \frac{-2(1+\beta \tau)}{\tau^{2}}\right) .
$$

Note that this limit point is identical to the point $(\alpha(0), \gamma(0))$ derived earlier in (2.8). For illustrative purposes, some curves $\bar{C}_{k}(\beta)$ are shown in Figure 5 for $\beta=1, \tau=1$, and $m=12$.

Now, one can prove very similar lemmas to those that have been proven in section 2.1. For example, the curves $\bar{C}_{k}(\beta)$ do not intersect, and they can be ordered in a natural way. We shall omit the (technical) proofs.

2.2.2. Stability region of the discrete scheme. Based on the continuity of the roots, one may state that the number of roots outside the unit disk is constant inside the regions of the $(\alpha, \gamma)$-space separated by the $\bar{C}_{k}(\beta)$-curves and the line $\bar{C}_{*}(\beta)$. It is easy to verify that all roots of (2.22) are in the unit disk when $\alpha=-|\beta|-1, \gamma=0$. Hence, we have the stability result given below. It states that the difference equation is asymptotically stable for the sets of parameters $(\alpha, \gamma)$ inside the region bounded by the line $\bar{C}_{*}(\beta)$ and the curve $\bar{C}_{0}(\beta)$ for the particular value of $m$. This is illustrated in Figure 6 , where the boundary curves $\bar{C}_{0}(\beta)$ are drawn for $\beta=0$ and for different $m$.

TheOREM 2.14. Difference equation (2.21) is asymptotically stable for $m=1$ iff

(a) $\alpha<\beta+\frac{2}{\tau}$ and

(b) $\alpha+\beta<-\gamma \tau$.

The equation is asymptotically stable for $m>1$ iff 


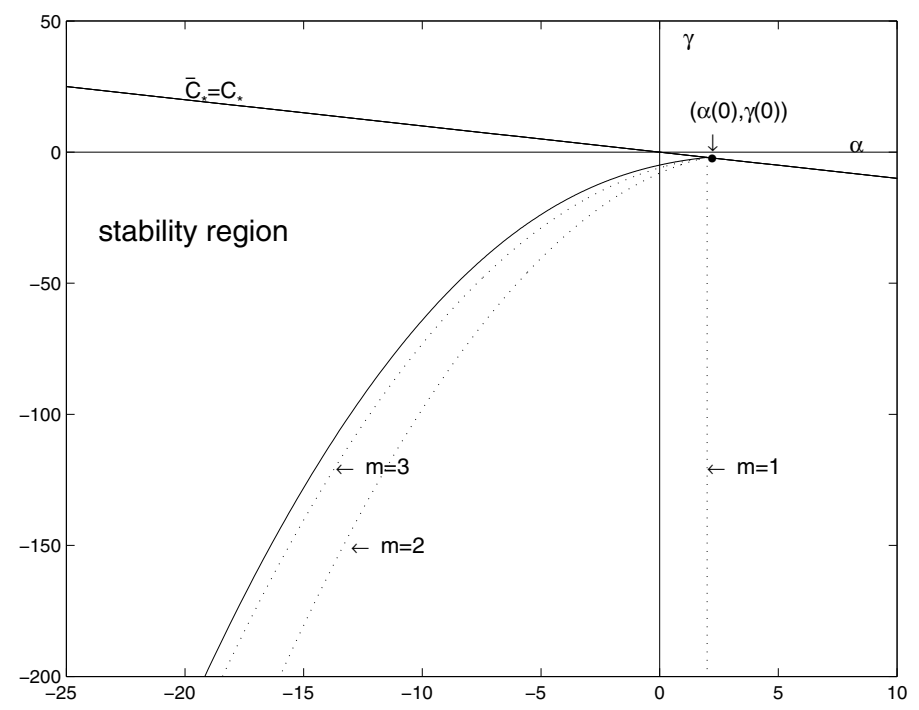

Fig. 6. Curve $C_{0}(\beta)$ (solid) and $\bar{C}_{0}(\beta)$-curves for different values of $m$ (dotted) for $\beta=0$.

(c) $\alpha<\beta+\frac{2}{\tau}$ and

(d) $(\alpha+\beta) \tau<-\gamma \tau^{2}<\frac{-2 m \sin \frac{\varphi}{m}\left(2 m \sin \frac{\varphi}{m}+\beta \tau \sin \varphi\left(1+\cos \frac{\varphi}{m}\right)\right)}{\left(1+\cos \frac{\varphi}{m}\right)^{2}(1-\cos \varphi)}$, where $\varphi$ is the root of $\alpha=\beta+\frac{2 m \sin \frac{\varphi}{m} \sin \varphi}{\tau\left(1+\cos \frac{\varphi}{m}\right)(1-\cos \varphi)}$ such that $\varphi \in(0,2 \pi)$.

Some more detailed features of the curve $\bar{C}_{0}(\beta)$ for the case $m>1$ will be required in our study of fully discrete delay PDE problems. The following analyses are similar to the ones in section 2.1.2, but technically somewhat more complicated. One can show that $\bar{\alpha}_{m}(\varphi)$ is strictly decreasing with respect to $\varphi \in \bar{J}_{0}$. The function $\bar{\gamma}_{m}(\varphi)$, on the other hand, for $\varphi \in \bar{J}_{0}$, satisfies

$$
\bar{\gamma}_{m}^{\prime}(\varphi)=\frac{2 m \beta \tau(m \sin \varphi-\sin m \varphi)}{\tau^{2}(1+\cos \varphi)(1-\cos m \varphi)}-\frac{4 m^{2} \sin \varphi(2-2 \cos m \varphi-m \sin \varphi \sin m \varphi)}{\tau^{2}(1+\cos \varphi)^{2}(1-\cos m \varphi)^{2}} .
$$

From this, one can derive that $\bar{\gamma}_{m}(\varphi)$ is strictly decreasing when $\beta \tau \leq \frac{m^{2}+2}{m^{2}-1}$. When $\beta \tau>\frac{m^{2}+2}{m^{2}-1}$, there exists a unique $\bar{\varphi}_{0} \in(0,2 \pi / m)$ such that

$$
\beta \tau=\frac{2 m \sin \bar{\varphi}_{0}\left(2-2 \cos m \bar{\varphi}_{0}-m \sin \bar{\varphi}_{0} \sin m \bar{\varphi}_{0}\right)}{\left(1-\cos m \bar{\varphi}_{0}\right)\left(1+\cos \bar{\varphi}_{0}\right)\left(m \sin \bar{\varphi}_{0}-\sin m \bar{\varphi}_{0}\right)}
$$

which gives

$$
\bar{\alpha}_{m}\left(\bar{\varphi}_{0}\right)=\frac{2 m \sin \bar{\varphi}_{0}\left(2-2 \cos m \bar{\varphi}_{0}-\sin ^{2} m \bar{\varphi}_{0}\right)}{\tau\left(1+\cos \bar{\varphi}_{0}\right)\left(1-\cos m \bar{\varphi}_{0}\right)\left(m \sin \bar{\varphi}_{0}-\sin m \bar{\varphi}_{0}\right)}>0 .
$$

Therefore, $\bar{C}_{0}(\beta)$ has exactly one extremum in the right half-plane. Let

$$
\bar{\alpha}_{0}= \begin{cases}\beta+\frac{2}{\tau} & \text { if } m=1, \\ \bar{\alpha}_{m}(0) & \text { if } \beta \tau \leq \frac{m^{2}+2}{m^{2}-1} \text { and } m>1, \\ \bar{\alpha}_{m}\left(\bar{\varphi}_{0}\right) & \text { otherwise }\end{cases}
$$


Then, for any given $\beta \in \mathbb{R}$ and $m>1, \bar{C}_{0}(\beta)$ is strictly monotonic in $(\alpha, \gamma)$-space when $\alpha \leq \bar{\alpha}_{0}$. In particular, $\bar{C}_{0}(\beta)$ is strictly monotonic in the left half of the $(\alpha, \gamma)$-plane. This leads to the following results, which will be used in the next section.

THEOREM 2.15. Suppose all roots $z$ of (2.22) lie inside the unit disk for given $\alpha, \beta, \gamma \in \mathbb{R}$, with $\alpha \leq \bar{\alpha}_{0}$ for $\bar{\alpha}_{0}$ defined in (2.26). Then for any $x \leq \alpha$, all the roots $z$ of the equation

$$
1-z^{-1}=\frac{h}{2}\left(1+z^{-1}\right)\left[x+\beta z^{-m}+\frac{\gamma h}{2}\left(1+z^{-1}\right) \sum_{j=0}^{m-1} z^{-j}\right]
$$

lie in the unit disk too.

Corollary 2.16. Suppose all roots $z$ of (2.22) lie in the unit disk for given $\alpha \leq 0, \beta, \gamma \in \mathbb{R}$. Then for any $x \leq \alpha$, all roots $z$ of (2.27) lie in the unit disk too.

2.2.3. Relation between the curves $C_{k}(\beta)$ and $\bar{C}_{k}(\beta)$. We are now in a position to derive some relations between the boundary locus curves $C_{k}(\beta)$ of the continuous problem and the curves $\bar{C}_{k}(\beta)$ of the discrete problem. These relations will lead immediately to the central result of the present section, which states that the stability region of the continuous problem is contained in the stability region of the discrete problem. The first lemma can be proven in a manner similar to Lemma 2.1 , by using elementary arithmetic.

Lemma 2.17. $C_{0}(\beta)$ and $\bar{C}_{0}(\beta)$ intersect only at the point $\left(\beta+\frac{2}{\tau}, \frac{-2(1+\beta \tau)}{\tau^{2}}\right)$.

Lemma 2.18. Curve $C_{0}(\beta)$ lies above curve $\bar{C}_{0}(\beta)$ in the $(\alpha, \gamma)$-plane for $m>1$.

Proof. The lemma follows from Lemma 2.17 combined with, for $m>1$,

$$
\alpha(\pi)=\bar{\alpha}_{m}\left(\frac{\pi}{m}\right)=\beta \quad \text { and } \quad \gamma(\pi)=\frac{-\pi^{2}}{2 \tau^{2}}>\frac{-2 m^{2} \sin ^{2} \frac{\pi}{m}}{\tau^{2}\left(1+\cos \frac{\pi}{m}\right)^{2}}=\bar{\gamma}_{m}\left(\frac{\pi}{m}\right) .
$$

THEOREM 2.19. The trapezium rule (2.21) preserves the asymptotic stability of problem (2.1) if $\alpha, \beta, \gamma$ satisfy the conditions of Theorem 2.7 .

Proof. Suppose $\alpha, \beta$, and $\gamma$ satisfy the conditions in Theorem 2.7. Then difference equation (2.21) is asymptotically stable. This follows immediately from Lemma 2.18 and from the fact that $C_{*}(\beta)=\bar{C}_{*}(\beta)$.

Specializing Theorem 2.20 to the case $\gamma=0$, one regains the stability result for the trapezium rule as obtained by Guglielmi in [11]. A further algebraic investigation reveals that for any fixed $\theta \in J_{k}$ with $k \geq 0$,

$$
\bar{\alpha}_{m}(\theta / m)-\alpha(\theta)=O\left(m^{-2}\right) \text { and } \bar{\gamma}_{m}(\theta / m)-\gamma(\theta)=O\left(m^{-2}\right) .
$$

With $O\left(m^{-2}\right)=O\left(h^{2}\right)$, the above result can be formulated as follows.

Theorem 2.20. Curve $\bar{C}_{k}(\beta)$ is an $O\left(h^{2}\right)$ approximation to curve $C_{k}(\beta)$.

Figure 7 shows the approximation of $\bar{C}_{k}(0)$ to $C_{k}(0)$ for $m=12$ and $\tau=1$.

3. Stability of delay partial differential equations. In this section, we will again consider analytical and discretized stability separately.

3.1. Stability of the continuous problem. Consider the two-dimensional parabolic functional-differential equation with both fixed and distributed delays,

$$
\frac{\partial u}{\partial t}=a\left(\frac{\partial^{2} u}{\partial x^{2}}+\frac{\partial^{2} u}{\partial y^{2}}\right)+b u(t-\tau, x, y)+c \int_{-\tau}^{0} u(t+s, x, y) d s,
$$




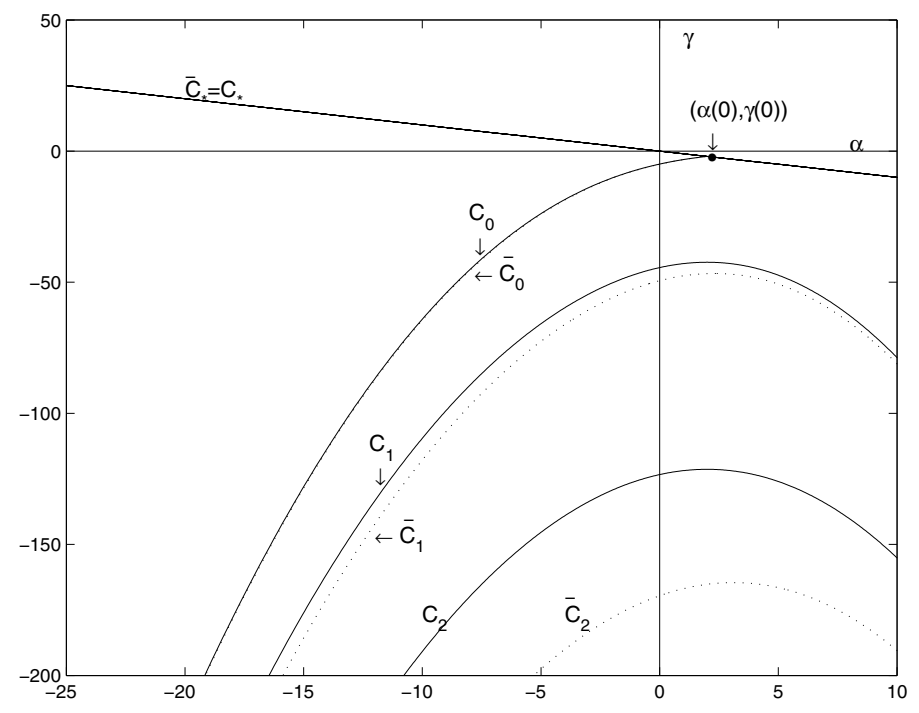

FIG. 7. The curves $\bar{C}_{k}(\beta)$ and $C_{k}(\beta)$ for $\beta=0, \tau=1$, and $m=12 . \bar{C}_{0}$ and $C_{0}$ almost overlap.

defined on the square domain $(x, y) \in \Omega=[0, L] \times[0, L]$ for $t>0$ and supplemented with initial and boundary conditions,

$$
\begin{aligned}
& u(t, x, y)=g(t, x, y), \quad t \in[-\tau, 0], \quad(x, y) \in \Omega, \\
& u(t, x, y)=0, \quad t>0, \quad(x, y) \in \partial \Omega .
\end{aligned}
$$

Here $a, b, c \in \mathbb{R} ; L$ and $\tau$ are positive constants. The characteristic equations of the above problem are given for $k, l=1,2, \ldots$ by

$$
\lambda=-a\left[\left(\frac{k \pi}{L}\right)^{2}+\left(\frac{l \pi}{L}\right)^{2}\right]+b \exp (-\lambda \tau)+c \int_{-\tau}^{0} \exp (\lambda s) d s
$$

see, e.g., [19, Chap. III]. The asymptotic stability of (3.1) is equivalent to the condition that all the roots of each of the equations (3.2) have negative real parts. This condition can be studied using the theory from section 2, setting the parameters $\alpha, \beta, \gamma$ as

$$
\alpha=-a\left[\left(\frac{k \pi}{L}\right)^{2}+\left(\frac{l \pi}{L}\right)^{2}\right], \beta=b, \gamma=c .
$$

The conditions of Theorem 2.7 can be reformulated for the present problem as

$$
\begin{array}{r}
-a\left[\left(\frac{k \pi}{L}\right)^{2}+\left(\frac{l \pi}{L}\right)^{2}\right]<b+\frac{2}{\tau} \quad \text { for } \quad k, l=1,2, \ldots, \\
-a\left[\left(\frac{k \pi}{L}\right)^{2}+\left(\frac{l \pi}{L}\right)^{2}\right]+b<-c \tau \quad \text { for } \quad k, l=1,2, \ldots, \\
-c \tau^{2}<\frac{\theta_{k l}\left(\theta_{k l}+b \tau \sin \theta_{k l}\right)}{1-\cos \theta_{k l}} \text { for } \quad k, l=1,2, \ldots,
\end{array}
$$


where $\theta_{k l}$ is the root of

$$
-a\left[\left(\frac{k \pi}{L}\right)^{2}+\left(\frac{l \pi}{L}\right)^{2}\right]=b+\frac{\theta_{k l} \sin \theta_{k l}}{\tau\left(1-\cos \theta_{k l}\right)}
$$

such that $\theta_{k l} \in(0,2 \pi)$. By using Corollary 2.10 one verifies the following result.

THEOREM 3.1. The zero solution of (3.1) is asymptotically stable iff

(a) $a \geq 0$,

(b) $-2 a \pi^{2} / L^{2}<b+2 / \tau$, and

(c) $\left(-2 a \pi^{2} / L^{2}+b\right) \tau<-c \tau^{2}<\frac{\theta(\theta+b \tau \sin \theta)}{1-\cos \theta}$,

where $\theta$ is the root of $-2 a \pi^{2} / L^{2}=b+\frac{\theta \sin \theta}{\tau(1-\cos \theta)}$ such that $\theta \in(0,2 \pi)$.

Proof. The "only if" part follows from (3.3)-(3.5) and the fact that (3.3) implies $a \geq 0$. The "if" part follows from Corollary 2.10, which implies that the stability is determined by the equation corresponding to the largest value of $\alpha$, i.e., the one corresponding to $k=l=1$.

Next we consider some special cases. First, we deal with the case of a parabolic PDE with a distributed delay, i.e., the case $b=0$,

$$
\frac{\partial u}{\partial t}=a\left(\frac{\partial^{2} u}{\partial x^{2}}+\frac{\partial^{2} u}{\partial y^{2}}\right)+c \int_{-\tau}^{0} u(t+s, x, y) d s
$$

A direct application of Theorem 3.1 to (3.7) yields the following result.

COROLlary 3.2. The zero solution of (3.7) is asymptotically stable iff

(a) $a \geq 0$ and

(b) $-2 \pi^{2} \tau a / L^{2}<-c \tau^{2}<\frac{\theta^{2}}{1-\cos \theta}$,

where $\theta$ is the root of $\theta \sin \theta=-2 \tau a \pi^{2}(1-\cos \theta) / L^{2}$ such that $\pi \leq \theta<2 \pi$.

For $L=\pi$ and $\tau=1$, the stability region of (3.7) in the (a,c)-plane is shown in the left panel of Figure 8. The right panel of that figure shows the stability region for $a=1 / 2$ and $c=-1$ in the $(\tau, L)$-plane.

The next example is a parabolic PDE with a fixed delay, i.e., with $c=0$,

$$
\frac{\partial u}{\partial t}=a\left(\frac{\partial^{2} u}{\partial x^{2}}+\frac{\partial^{2} u}{\partial y^{2}}\right)+b u(t-\tau, x, y) .
$$

A similar application of the general theorem leads to the following corollary.

COROLlary 3.3. The zero solution of (3.8) is asymptotically stable iff

(a) $a \geq 0$ and

(b) $-2 \pi^{2} a / L^{2}<-b<\frac{\theta}{\tau \sin \theta}$,

where $\theta$ is the root of $\theta \cos \theta=-2 \tau a \pi^{2} \sin \theta / L^{2}$ such that $\pi / 2 \leq \theta<\pi$.

For $L=\pi$ and $\tau=1$, the stability region of the zero solution to (3.8) is shown in the $(a, b)$-plane in the left panel of Figure 9 . The right panel of that figure shows the stability region in the $(\tau, L)$-plane for fixed $a=1 / 2$ and $b=-1$.

3.2. Stability of the semidiscrete PDE problem. We discretize the spatial variables $(x, y)$ into $(N+2) \times(N+2)$ discrete values $(N>0)$ with a constant stepsize $\Delta x=\Delta y=L /(N+1)$, so that $x_{i}=i \Delta x, y_{j}=j \Delta y$ for $i, j=0,1,2, \ldots, N+1$. Using the standard central difference operator to approximate the Laplacian, we obtain a 

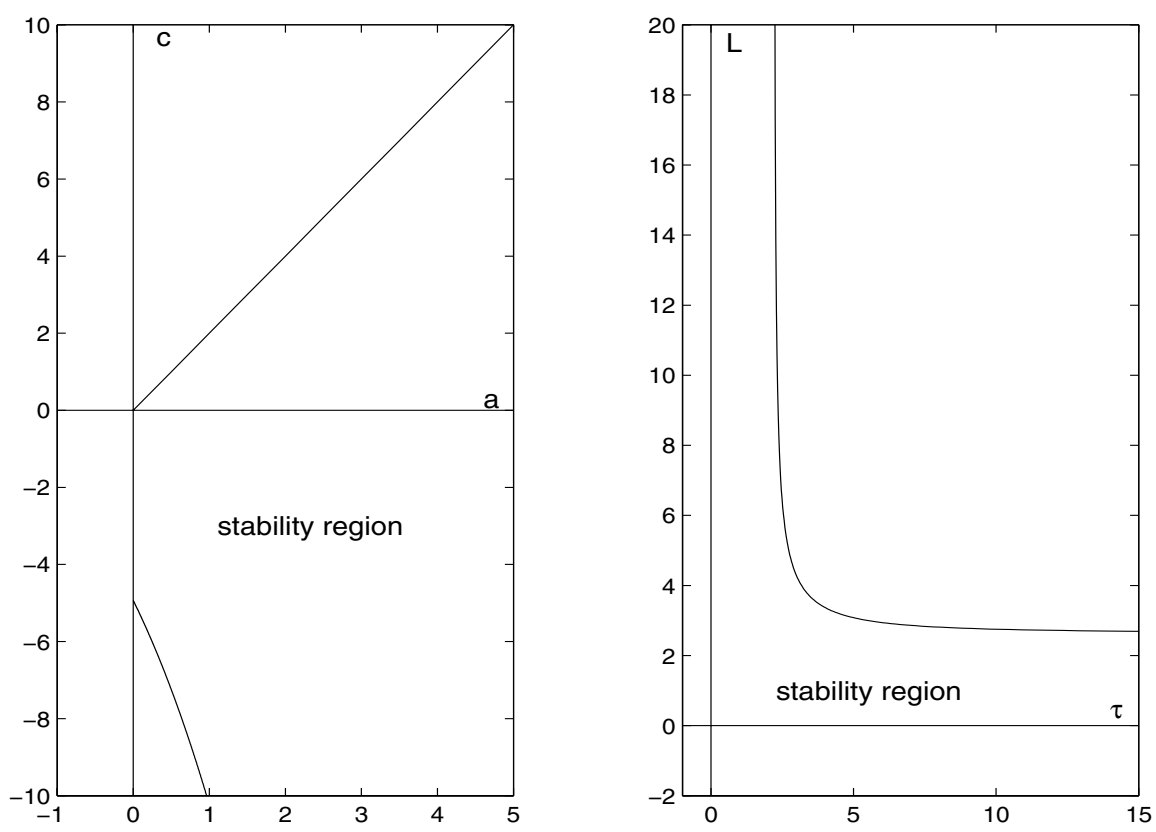

FIG. 8. Stability region of distributed delay PDE (3.7). Left: $(a, c)$-plane with $L=\pi$ and $\tau=1$. Right: $(\tau, L)$-plane with $a=1 / 2$ and $c=-1$.
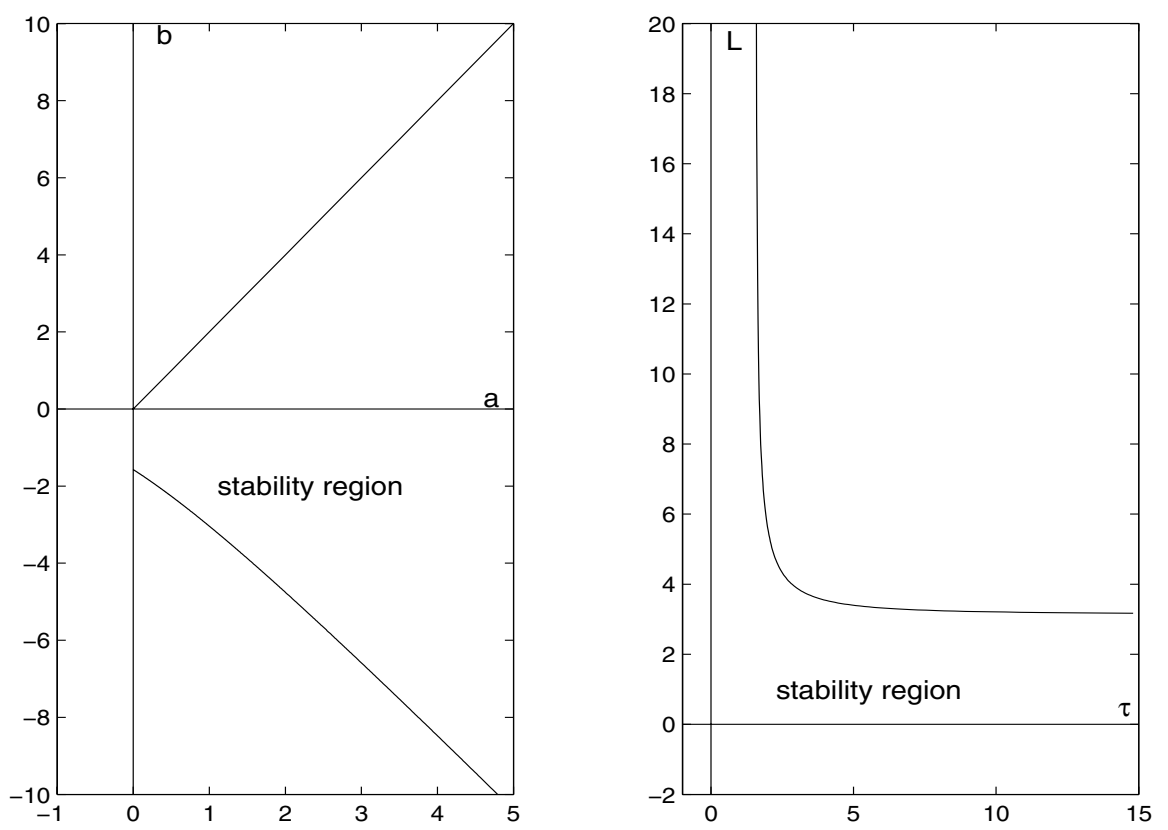

FIG. 9. Stability region of fixed delay PDE (3.8). Left: $(a, b)$-plane with $L=\pi$ and $\tau=1$. Right: $(\tau, L)$-plane with $a=1 / 2$ and $b=-1$. 
system of delay ODEs of the form

$$
\begin{aligned}
u_{i j}^{\prime}(t)= & a\left(\frac{u_{i+1, j}(t)+u_{i-1, j}(t)+u_{i, j+1}(t)+u_{i, j-1}(t)-4 u_{i j}(t)}{\Delta x^{2}}\right) \\
& +b u_{i j}(t-\tau)+c \int_{-\tau}^{0} u_{i j}(t+s) d s, \quad i, j=1, \ldots, N
\end{aligned}
$$

where $u_{i j}(t)$ denotes an approximation to $u\left(t, x_{i}, y_{j}\right)$. Let

$$
U(t)=\left(u_{11}(t), \ldots, u_{1 N}(t), u_{21}(t), \ldots, u_{2 N}(t), \ldots \ldots, u_{N 1}(t), \ldots, u_{N N}(t)\right)^{T} .
$$

Considering the homogeneous Dirichlet conditions and the fact that $\Delta x=\Delta y$, one can write the semidiscrete system compactly as

$$
U^{\prime}(t)=\frac{a(N+1)^{2}}{L^{2}}\left(A \otimes I_{N}+I_{N} \otimes A\right) U(t)+b U(t-\tau)+c \int_{-\tau}^{0} U(t+s) d s,
$$

where $I_{N}$ stands for the $N \times N$ identity matrix and

$$
A=\left[\begin{array}{ccccc}
-2 & 1 & & & \\
1 & -2 & \ddots & & \\
& \ddots & \ddots & \ddots & \\
& & \ddots & \ddots & 1 \\
& & & 1 & -2
\end{array}\right]_{N \times N}
$$

The asymptotic stability of the zero solution to (3.10) is equivalent to the condition that all the roots of the characteristic equation

$\operatorname{det}\left(\left(\lambda-b \exp (-\lambda \tau)-c \int_{-\tau}^{0} \exp (\lambda s) d s\right) I_{N^{2}}-\frac{a(N+1)^{2}}{L^{2}}\left(A \otimes I_{N}+I_{N} \otimes A\right)\right)=0$

have negative real parts. The eigenvalues of $A$ are given by

$$
\lambda_{i}^{A}=-4 \sin ^{2} \frac{i \pi}{2(N+1)}, \quad i=1, \ldots, N .
$$

Therefore, the eigenvalues of the matrix

$$
\frac{(N+1)^{2}}{L^{2}}\left(A \otimes I_{N}+I_{N} \otimes A\right)
$$

are given by

$$
\lambda_{i j}=\frac{-4(N+1)^{2}}{L^{2}}\left(\sin ^{2} \frac{i \pi}{2(N+1)}+\sin ^{2} \frac{j \pi}{2(N+1)}\right), i, j=1, \ldots, N .
$$

As a consequence, the asymptotic stability of system (3.10) is equivalent to the condition that all the roots of each of the equations

$$
\lambda-a \lambda_{i j}-b \exp (-\lambda \tau)-c \int_{-\tau}^{0} \exp (\lambda s) d s=0, \quad i, j=1, \ldots, N,
$$

have negative real parts. A direct application of Corollary 2.10 reveals the conditions for stability. 
TheOREM 3.4. Assume $a \geq 0$. Then the zero solution of the semidiscrete delay PDE (3.9) is asymptotically stable iff

(a) $a \lambda_{11}<b+2 / \tau$ and

(b) $\left(a \lambda_{11}+b\right) \tau<-c \tau^{2}<\frac{\theta(\theta+b \tau \sin \theta)}{1-\cos \theta}$, where $\theta$ is the root of $a \lambda_{11}=b+\frac{\theta \sin \theta}{\tau(1-\cos \theta)}, \theta \in(0,2 \pi)$, and $\lambda_{11}$ is defined in (3.12).

Proof. Based on Corollary 2.10, one can see that the stability is determined by the equation corresponding to the largest value of $\alpha$, i.e., the eigenvalue $\lambda_{11}$.

The semidiscretization of delay PDE (3.8) leads to a set of delay ODEs

$$
u_{i j}^{\prime}=a\left(\frac{u_{i+1, j}+u_{i-1, j}+u_{i, j+1}+u_{i, j-1}-4 u_{i j}}{\Delta x^{2}}\right)+b u_{i j}(t-\tau)
$$

for $i, j=1, \ldots, N$. Analysis of this set of equations yields the next result.

Corollary 3.5. Assume $a \geq 0$. Then the zero solution of the semidiscrete delay PDE (3.14) is asymptotically stable iff

$$
a \lambda_{11}<-b<\frac{\theta}{\tau \sin \theta},
$$

where $\theta$ is the root of $\theta \cos \theta=a \lambda_{11} \tau \sin \theta$ such that $\pi / 2 \leq \theta<\pi$ and $\lambda_{11}$ is defined in (3.12).

The semidiscretization of the distributed delay PDE (3.7) gives

$$
u_{i j}^{\prime}=a\left(\frac{u_{i+1, j}+u_{i-1, j}+u_{i, j+1}+u_{i, j-1}-4 u_{i j}}{\Delta x^{2}}\right)+c \int_{-\tau}^{0} u_{i j}(t+s) d s
$$

for $i, j=1, \ldots, N$. A direct application of Theorem 3.4 leads to the next corollary.

Corollary 3.6. Assume $a \geq 0$. Then the zero solution of the semidiscrete delay PDE (3.15) is asymptotically stable iff

$$
a \lambda_{11} \tau<-c \tau^{2}<\frac{\theta^{2}}{1-\cos \theta}
$$

where $\theta$ is the root of $\theta \sin \theta=a \lambda_{11} \tau(1-\cos \theta)$ such that $\pi \leq \theta<2 \pi$.

It is interesting to compare the stability region of the original PDE with that of the semidiscrete set of ODEs. The following theorem shows that when $a>0$, the stability region of the latter is smaller than the stability region of the former. This is different from the case of PDEs without delays. Here we do not draw the pictures of these stability regions because they can barely be distinguished from their counterparts in the continuous case. Besides, both regions converge to each other when $\Delta x \rightarrow 0$.

TheOREM 3.7. Assume $a>0$. Then the asymptotic stability region of (3.9) is a subset of the asymptotic stability region of (3.1).

Proof. Based on Corollary 2.10, one can see that the stability of (3.9) is determined by (3.13) with $i=j=1$ and the stability of (3.1) is determined by (3.2) with $k=l=1$. A repeated application of Corollary 2.10 combined with $-2 \pi^{2} / L^{2}<\lambda_{11}<0$ and $a>0$ leads to the conclusion. 
3.3. Stability of the fully discrete PDE problem. An application of the trapezium rule to (3.9) leads to the scheme

$$
\begin{aligned}
\frac{u_{i j}^{n+1}-u_{i j}^{n}}{\Delta t}= & \frac{a}{2}\left(\frac{u_{i+1, j}^{n+1}+u_{i-1, j}^{n+1}+u_{i, j+1}^{n+1}+u_{i, j-1}^{n+1}-4 u_{i j}^{n+1}}{\Delta x^{2}}\right) \\
& +\frac{b}{2} u_{i j}^{n+1-m}+\frac{c \Delta t}{4}\left(\sum_{k=0}^{m-1} u_{i j}^{n+1-k}+\sum_{k=1}^{m} u_{i j}^{n+1-k}\right) \\
& +\frac{a}{2}\left(\frac{u_{i+1, j}^{n}+u_{i-1, j}^{n}+u_{i, j+1}^{n}+u_{i, j-1}^{n}-4 u_{i j}^{n}}{\Delta x^{2}}\right) \\
& +\frac{b}{2} u_{i j}^{n-m}+\frac{c \Delta t}{4}\left(\sum_{k=0}^{m-1} u_{i j}^{n-k}+\sum_{k=1}^{m} u_{i j}^{n-k}\right)
\end{aligned}
$$

for $i, j=1,2, \ldots, N$; the time stepsize is given by $\Delta t=\tau / m$, and each $u_{i j}^{n}$ denotes an approximation to $u(n \Delta t, i \Delta x, j \Delta y)$. An analysis similar to the one leading to Theorem 3.4 in combination with Theorem 2.14 and Corollary 2.16 leads to the next theorem.

Theorem 3.8. Assume $a \geq 0$. Let $\lambda_{11}$ be defined by (3.12). When $m=1$, the difference equation (3.16) is asymptotically stable iff

(a) $a \lambda_{11}<b+\frac{2}{\tau}$ and

(b) $a \lambda_{11}+b<-c \tau$.

When $m>1$, the difference equation (3.16) is asymptotically stable iff

(c) $a \lambda_{11}<b+\frac{2}{\tau}$ and

(d) $\left(a \lambda_{11}+b\right) \tau<-c \tau^{2}<\frac{-2 m \sin \frac{\varphi}{m}\left(2 m \sin \frac{\varphi}{m}+b \tau \sin \varphi\left(1+\cos \frac{\varphi}{m}\right)\right)}{\left(1+\cos \frac{\varphi}{m}\right)^{2}(1-\cos \varphi)}$,

where $\varphi$ is the root of $a \lambda_{11}=b+\frac{2 m \sin \frac{\varphi}{m} \sin \varphi}{\tau\left(1+\cos \frac{\varphi}{m}\right)(1-\cos \varphi)}$ such that $\varphi \in(0,2 \pi)$.

This scheme fully preserves the stability of ODE system (3.9). It is easily seen, however, that the fully discrete scheme (3.16) cannot completely preserve the asymptotic stability of the underlying PDE system (3.1). In fact, we have the following general result.

TheOREm 3.9. Any consistent time discretization scheme applied to the spatial discretization (3.9) cannot completely preserve the asymptotic stability of (3.1).

Proof. We consider parameters $a, b, c \in \mathbb{R}$ such that

$$
a \lambda_{11}+b+c \tau=0
$$

and show that there always exist such parameters that are outside the fully discrete stability region yet inside the fully continuous stability region.

Let $V \in \mathbb{R}^{N^{2}}$ be the eigenvector corresponding to the eigenvalue $\lambda_{11}$ of the matrix (3.11). Consider the initial value problem (3.10) with the initial value condition $U(t)=V, t \in[-\tau, 0]$. Then, with any $a, b$, and $c$ satisfying (3.17), the exact solution of (3.9) on interval $[0,+\infty)$ is $U(t)=V$. Therefore, the numerical solution of any consistent scheme is always $V$ too. Hence, every $(a, b, c)$ determined by (3.17) is always out of the asymptotic stability region of the fully discrete scheme. On the other hand, analogously to the proof of Theorem 3.7, the fact

$$
0>\lambda_{11}>\frac{-2 \pi^{2}}{L^{2}}
$$

implies that there exists a part of the sets of parameters $(a, b, c)$ determined by $(3.17)$, which belongs to the stability region of (3.1). This completes the proof. 
TABLE 1

The norm of the numerical solution of (4.1) at $T=1200$ as a function of $\Delta t$ and $\Delta x$ for $a=8, b=-8.4772, c=0$.

\begin{tabular}{|c|c|c|c|c|}
\hline & $\Delta x=\pi / 9$ & $\Delta x=\pi / 17$ & $\Delta x=\pi / 33$ & $\Delta x=\pi / 65$ \\
\hline$\Delta t=1 / 2$ & $3.1127 \mathrm{E}-05$ & $3.8150 \mathrm{E}-08$ & $1.1394 \mathrm{E}-04$ & 0.0111 \\
\hline$\Delta t=1 / 4$ & 362.7204 & 0.2301 & 0.0459 & 0.0274 \\
\hline$\Delta t=1 / 8$ & 405.9670 & 8.0659 & 0.3400 & 0.0630 \\
\hline$\Delta t=1 / 16$ & $1.2529 \mathrm{E}+04$ & 23.2885 & 2.1096 & 0.9810 \\
\hline
\end{tabular}

Remark 3.1. The above proof shows that a reduction of the time stepsize cannot improve the stability of the scheme. No matter how small $\Delta t$, one can always find a set of parameters for which the PDE is asymptotically stable, while the fully discrete system is not asymptotically stable. This instability typically happens when the set of parameters is located near the boundary of the analytical stability region, and it can lead to computational problems when performing long-time integration (see numerical experiments in section 4). However, we can appropriately improve the stability by reducing the spatial stepsize. An alternative choice is to construct new spatial discretizations such that the semidiscrete system completely preserves the asymptotic stability of the original PDE. We will give a more detailed discussion and an example in the next section.

4. Numerical examples. For simplicity, we consider the one-dimensional equation on $x \in[0, \pi]$, and we set the delay $\tau=1$,

$$
\frac{\partial}{\partial t} u(t, x)=a \frac{\partial^{2}}{\partial x^{2}} u(t, x)+b u(t-1, x)+c \int_{-1}^{0} u(t+s, x) d s,
$$

with $u(t, x) \equiv 1, t \leq 0, x \in(0, \pi)$, and $u(t, x)=0, t>0, x \in\{0, \pi\}$. First, we will present some results that illustrate the theory developed in this paper. Next, in section 4.2 , we will study numerically some cases that have not been analyzed in the preceding sections: a different spatial discretization scheme, a high-order RungeKutta time-integration scheme, and the use of nonconstrained timesteps.

4.1. Trapezium rule with the standard central difference. In this subsection we present some numerical examples illustrating the difference between the stability regions of the continuous and the discretized systems. The problem is discretized with central differences in space with space stepsize $\Delta x=\pi /(N+1)$ and with the trapezium rule in time, with time stepsize $\Delta t=1 / \mathrm{m}$. We consider the norm of the numerical solution at time-point $T=1200$ as a function of $\Delta t$ and $\Delta x$. Here we use the weighted discrete $L_{2}$-norm to measure the numerical solution:

$$
\|u\|=\sqrt{\sum_{j=1}^{N}\left(u_{j}\right)^{2} \Delta x}, \quad u=\left(u_{1}, u_{2}, \ldots, u_{N}\right)^{T} \in \mathbb{R}^{N} .
$$

First we consider a fixed delay PDE problem with parameters $a=8, b=-8.4772$, and $c=0$. From Theorem 3.1 it follows that the analytical solution of (4.1) is asymptotically stable, whereas, by Theorem 3.4, the analytical solution of the relevant semidiscrete delay PDE (3.9) is not. The norm of the numerical solution is given in Table 1. The column of values for $\Delta x=\pi / 17$, i.e., $N=16$, will be explained by means of Figure 10 (left). The row of values for $\Delta t=1 / 8$, i.e., $m=8$, will be 

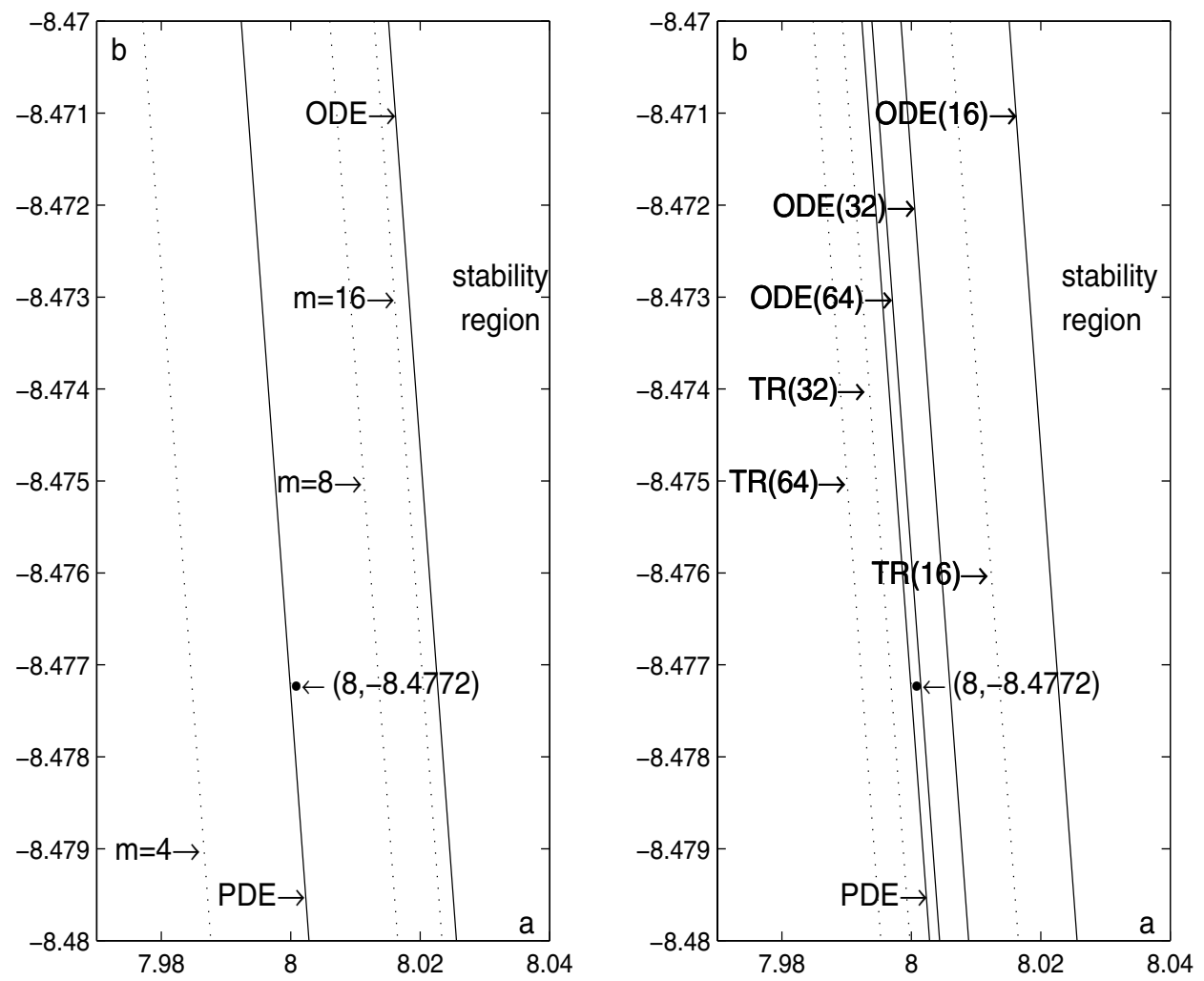

FIG. 10. Local boundaries of the stability regions of (3.1) and the discrete systems with $c=0$. The left panel depicts those for $N=16$ and for different $m$ (dotted). The right panel depicts those for $m=8$ and for different $N$ (solid: semidiscrete systems; dotted: fully discrete systems).

explained by means of Figure 10 (right). Next, we also consider a distributed delay PDE problem with parameters $a=10, b=0, c=9.999$. From Theorem 3.1 it follows that the analytical solution is asymptotically stable too. The norm of the numerical solution is given in Table 2 .

These numerical examples confirm our theoretical findings. In the case of delay PDEs, the trapezium rule can preserve only the stability region of the semidiscrete system, which is smaller than that of the original PDE. The reduction of the time stepsize leads to a better approximation to the stability region of the semidiscrete system so that the stability region of the fully discrete scheme is further reduced. This is illustrated in Figure 10, where the local boundary curves of the stability regions of the scheme for $c=0$ and $N=16$ and for different $m$ are shown in the left panel. Obviously, the point $(8,-8.4772)$ is located in the stability region of PDE but outside the stability region of the relevant semidiscrete system. When $m=4$ (i.e., $\Delta t=1 / 4)$, it is also in the stability region of the fully discrete scheme. When $m=8$ or 16, however, it is out of the stability region of the scheme. From the picture it is obvious to see that a smaller time stepsize leads to a smaller stability region. This is in accordance with the numerical results in Table 1. However, the reduction of the spatial stepsize is useful for improving the stability: it leads to a better approximation to the stability region of the original PDE. This is shown in the right panel, where the 
TABLE 2

The norm of the numerical solution of (4.1) at $T=1200$ as a function of $\Delta t$ and $\Delta x$ for $a=10, b=0, c=9.999$.

\begin{tabular}{|c|c|c|c|c|}
\hline & $\Delta x=\pi / 9$ & $\Delta x=\pi / 17$ & $\Delta x=\pi / 33$ & $\Delta x=\pi / 65$ \\
\hline$\Delta t=1 / 8$ & $8.6918 \mathrm{E}+08$ & 387.0720 & 5.9148 & 1.9281 \\
\hline$\Delta t=1 / 16$ & $8.6868 \mathrm{E}+08$ & 387.0532 & 5.9148 & 1.9281 \\
\hline
\end{tabular}

local boundary curves of the stability regions of the semidiscrete and fully discrete $(m=8)$ systems for different $N$ are shown.

The results of Table 2 are different from those in Table 1 . This is because we selected a point $(a, c)$ near the boundary curve $C_{*}$, which depends only on the spatial stepsize but which is independent of the time stepsize. The changes of the time stepsize do not affect the stability. A spatial stepsize reduction, however, leads to a bigger stability region of the scheme, as is illustrated in Table 2.

4.2. Numerical study of alternative approaches. Although the preceding theoretical analysis is strictly confined to a specialized model problem, it already provides some insight into the stability behavior of delay PDEs. In this subsection, we consider some alternative approaches that are not strictly covered by the theory of the preceding sections. The first one is concerned with spatial discretization. In order to preserve the stability of the model PDE, we introduce an alternative to the standard central difference scheme. In particular, we have

$$
\frac{\partial^{2} u}{\partial x^{2}}+\frac{\partial^{2} u}{\partial y^{2}} \approx \frac{\pi^{2}\left(u_{i+1, j}(t)+u_{i-1, j}(t)+u_{i, j+1}(t)+u_{i, j-1}(t)-4 u_{i j}(t)\right)}{4 L^{2} \sin ^{2} \frac{\pi \Delta x}{2 L}},
$$

which is also consistent with order 2 in space. An analysis of the eigenvalues of the corresponding matrix (3.11) shows that the largest eigenvalue in magnitude is now identical to the largest eigenvalue of the diffusion operator on $\Omega$. Considering also the fact that the trapezium rule preserves the stability of the semidiscrete system, we may state the following result.

TheOREm 4.1. Assume $a>0$. Then the stability region of the semidiscrete system based on (4.2) is exactly the same as the stability region of the original PDE (3.1). Furthermore, the corresponding fully discrete scheme based on the trapezium rule also preserves the asymptotic stability of (3.1).

For the one-dimensional PDE (4.1), some numerical results corresponding to the spatial discretization (4.2) are given in Tables 3 and 4. Comparing these results with those in Tables 1 and 2, one can see that a slight change of scheme has resulted in a very different outcome. The instability due to the spatial discretization has indeed disappeared. So far it is unclear, however, whether the above adaptation can also be applied successfully to equations that are more general than the model equation. This is a topic which certainly merits further investigation.

Remark 4.1. Note that the above results and the results in section 4.1 cannot be explained by the existing theory of delay-independent stability. In the nondistributed delay case considered here, i.e., with $c=0$, the delay-independent stability condition for (4.1) is given by the condition $-a \leq b<a$. This condition is not satisfied for the problem solved in the numerical experiment. The delay-independent analysis excludes a large class of actually stable and interesting problems. This limitation is the main reason for studying delay-dependent stability. The latter does provide a much more detailed picture for when solutions of delay problems are stable or not. 
TABLE 3

The norm of the numerical solution based on the trapezium rule together with the discretization (4.2) at $T=1200$ as a function of $\Delta t$ and $\Delta x$ for $a=8, b=-8.4772, c=0$.

\begin{tabular}{|c|c|c|c|}
\hline & $\Delta x=\pi / 9$ & $\Delta x=\pi / 17$ & $\Delta x=\pi / 33$ \\
\hline$\Delta t=1 / 2$ & $3.7108 \mathrm{E}-09$ & $3.7452 \mathrm{E}-09$ & $1.1445 \mathrm{E}-04$ \\
\hline$\Delta t=1 / 4$ & 0.0225 & 0.0227 & 0.0227 \\
\hline$\Delta t=1 / 8$ & 0.0128 & 0.0129 & 0.0129 \\
\hline$\Delta t=1 / 16$ & 0.7270 & 0.7337 & 0.7356 \\
\hline
\end{tabular}

TABLE 4

The norm of the numerical solution based on the trapezium rule together with the discretization (4.2) at $T=1200$ as a function of $\Delta t$ and $\Delta x$ for $a=10, b=0, c=9.999$.

\begin{tabular}{|c|c|c|c|}
\hline & $\Delta x=\pi / 9$ & $\Delta x=\pi / 17$ & $\Delta x=\pi / 33$ \\
\hline$\Delta t=1 / 8$ & 1.2899 & 1.3018 & 1.3052 \\
\hline$\Delta t=1 / 16$ & 1.2899 & 1.3018 & 1.3052 \\
\hline
\end{tabular}

TABLE 5

The norm of the numerical solution based on the 2-stage Gauss method together with the standard central difference at $T=1200$ as a function of $\Delta t$ and $\Delta x$ for $a=8, b=-8.4772, c=0$.

\begin{tabular}{|c|c|c|c|}
\hline & $\Delta x=\pi / 9$ & $\Delta x=\pi / 17$ & $\Delta x=\pi / 33$ \\
\hline$\Delta t=1 / 2$ & $2.0885 \mathrm{E}+04$ & 15.6670 & 2.49729 \\
\hline$\Delta t=1 / 4$ & $1.0980 \mathrm{E}+04$ & 17.2852 & 0.0863 \\
\hline$\Delta t=1 / 8$ & $1.4990 \mathrm{E}+04$ & 15.1072 & 0.5122 \\
\hline$\Delta t=1 / 16$ & $1.5250 \mathrm{E}+04$ & 14.9568 & 0.5402 \\
\hline
\end{tabular}

TABLE 6

The norm of the numerical solution based on the 2-stage Gauss method together with the discretization (4.2) at $T=1200$ as a function of $\Delta t$ and $\Delta x$ for $a=8, b=-8.4772, c=0$.

\begin{tabular}{|c|c|c|c|}
\hline & $\Delta x=\pi / 9$ & $\Delta x=\pi / 17$ & $\Delta x=\pi / 33$ \\
\hline$\Delta t=1 / 2$ & 1.1305 & 1.1410 & 1.1440 \\
\hline$\Delta t=1 / 4$ & 0.4644 & 0.4687 & 0.4700 \\
\hline$\Delta t=1 / 8$ & 0.6712 & 0.6774 & 0.6792 \\
\hline$\Delta t=1 / 16$ & 0.6846 & 0.6910 & 0.6928 \\
\hline
\end{tabular}

Next, we consider a time discretization scheme of high order. We select the 2-stage Gauss method of order 4, which can be represented by the Butcher tableau

$$
\begin{array}{c|cc}
1 / 2-\sqrt{3} / 6 & 1 / 4 & 1 / 4-\sqrt{3} / 6 \\
1 / 2+\sqrt{3} / 6 & 1 / 4+\sqrt{3} / 6 & 1 / 4 \\
\hline & 1 / 2 & 1 / 2
\end{array} .
$$

The corresponding numerical results are given in Tables 5 and 6 for the two different spatial discretizations. These results suggest that the method possesses a stability property similar to the trapezium rule. For a rigorous theoretical analysis of the stability of high-order Runge-Kutta methods for (2.1), we refer to the paper [15], which was recently finished by the same authors.

Finally, we present a numerical experiment with a constant time stepsize, which is not a submultiple of the delay. Let $\tau=(m-\delta) \Delta t$ with integer $m$ and $\delta \in[0,1)$. 
TABLE 7

The norm of the numerical solution with a nonconstrained grid $(\delta=0.1)$ and the standard central difference at $T=1200$ as a function of $\Delta t$ and $\Delta x$ for $a=8, b=-8.4772, c=0$.

\begin{tabular}{|c|c|c|c|}
\hline & $\Delta x=\pi / 9$ & $\Delta x=\pi / 17$ & $\Delta x=\pi / 33$ \\
\hline$\Delta t=1 / 2$ & $3.4529 \mathrm{E}-33$ & $1.6056 \mathrm{E}-10$ & $2.0712 \mathrm{E}-04$ \\
\hline$\Delta t=1 / 4$ & $8.1822 \mathrm{E}-09$ & $6.6577 \mathrm{E}-12$ & $1.0856 \mathrm{E}-11$ \\
\hline$\Delta t=1 / 8$ & 21.5583 & 0.0240 & 0.0030 \\
\hline$\Delta t=1 / 16$ & $3.4863 \mathrm{E}+03$ & 5.2564 & 0.5326 \\
\hline
\end{tabular}

TABLE 8

The norm of the numerical solution with a nonconstrained grid $(\delta=0.1)$ and the discretization (4.2) at $T=1200$ as a function of $\Delta t$ and $\Delta x$ for $a=8, b=-8.4772, c=0$.

\begin{tabular}{|c|c|c|c|}
\hline & $\Delta x=\pi / 9$ & $\Delta x=\pi / 17$ & $\Delta x=\pi / 33$ \\
\hline$\Delta t=1 / 2$ & $7.0016 \mathrm{E}-33$ & $1.6934 \mathrm{E}-10$ & $2.0795 \mathrm{E}-04$ \\
\hline$\Delta t=1 / 4$ & $3.4768 \mathrm{E}-13$ & $3.5090 \mathrm{E}-13$ & $1.1032 \mathrm{E}-11$ \\
\hline$\Delta t=1 / 8$ & 0.0012 & 0.0012 & 0.0013 \\
\hline$\Delta t=1 / 16$ & 0.1986 & 0.2004 & 0.2009 \\
\hline
\end{tabular}

When $c=0$, we consider the fully discrete scheme for (4.1),

$$
\begin{aligned}
\frac{u_{j}^{n+1}-u_{j}^{n}}{\Delta t}= & \frac{a}{2}\left(\frac{u_{j+1}^{n+1}+u_{j-1}^{n+1}-2 u_{j}^{n+1}}{\Delta x^{2}}\right)+\frac{a}{2}\left(\frac{u_{j+1}^{n}+u_{j-1}^{n}-2 u_{j}^{n}}{\Delta x^{2}}\right) \\
& +\frac{b}{2}\left((1-\delta) u_{j}^{n-m}+u_{j}^{n+1-m}+\delta u_{j}^{n+2-m}\right)
\end{aligned}
$$

for $j=1,2, \ldots, N$, where we use the linear interpolation $\delta u_{j}^{n+1-m}+(1-\delta) u_{j}^{n-m}$ to approximate the delay argument $u_{j}\left(t_{n}-\tau\right)=u_{j}\left(t_{n-m}+\delta \Delta t\right)$. An alternative is to replace the standard central difference by the spatial discretization (4.2). The corresponding numerical results for $\delta=0.1$ are given in Tables 7 and 8 . The scheme with a nonconstrained grid appears to have a stability property similar to the scheme with a constrained grid (i.e., $\delta=0$ ). However, a rigorous theoretical analysis that supports this observation is missing (and outside the scope of the present study).

5. Concluding remarks. In this work novel stability properties of numerical methods for differential equations with both fixed and distributed delays have been introduced. A complete stability analysis has been accomplished for the trapezium rule with respect to the real coefficient test problems of both ordinary and partial differential equations. A positive result is that the time discretization based on the trapezium rule can preserve the asymptotic stability of underlying ODE test problems. A negative result is that the spatial discretization based on standard central difference unavoidably leads to a reduction of the asymptotic stability region of the corresponding PDE problem. Any consistent full discretization based on central difference cannot completely preserve the asymptotic stability of the original delay PDE.

Acknowledgments. The authors are indebted to the anonymous referees for the careful reading of a preliminary version of the manuscript and their valuable suggestions and comments. The authors also thank Dr. Tatyana Luzyanina for many interesting discussions. 


\section{REFERENCES}

[1] A. N. Al Mutib, Stability properties of numerical methods for solving delay differential equations, J. Comput. Appl. Math., 10 (1984), pp. 71-79.

[2] C. T. H. BAKer, Retarded differential equations, J. Comput. Appl. Math., 125 (2000), pp. 309-335.

[3] C. T. H. BAKer, A perspective on the numerical treatment of Volterra equations, J. Comput. Appl. Math., 125 (2000), pp. 217-249.

[4] C. T. H. BAKer AND N. J. Ford, Stability properties of a scheme for the approximate solution of a delay-integro-differential equation, Appl. Numer. Math., 9 (1992), pp. 357-370.

[5] C. T. H. BaKer And C. A. H. PAUL, Computing stability regions-Runge-Kutta methods for delay differential equations, IMA J. Numer. Anal., 14 (1994), pp. 347-362.

[6] A. Bellen and M. Zennaro, Numerical Methods for Delay Differential Equations, Oxford University Press, Oxford, 2003.

[7] M. Calvo and T. Grande, On the asymptotic stability of $\Theta$-methods for delay differential equations, Numer. Math, 54 (1988), pp. 257-269.

[8] C. W. CRYer, Highly stable multistep methods for retarded differential equations, SIAM J. Numer. Anal., 11 (1974), pp. 788-797.

[9] O. Diekmann, S. A. Van Gils, S. M. Verduyn Lunel, and H. -O. Walther, Delay Equations. Functional, Complex, and Nonlinear Analysis, Appl. Math. Sci. 110, Springer-Verlag, Berlin, 1995.

[10] N. Guglielmi, On the asymptotic stability properties of Runge-Kutta methods for delay differential equations, Numer. Math, 77 (1997), pp. 467-485.

[11] N. Guglielmi, Delay dependent stability regions of $\Theta$-methods for delay differential equations, IMA J. Numer. Anal., 18 (1998), pp. 399-418.

[12] N. Guglielmi, Asymptotic stability barriers for natural Runge-Kutta processes for delay equations, SIAM J Numer. Anal., 39 (2001), pp. 763-783.

[13] N. Guglielmi And E. Hairer, Order stars and stability for delay differential equations, Numer. Math., 83 (1999), pp. 371-383.

[14] N. Guglielmi and E. Hairer, Geometric proofs of numerical stability for delay equations, IMA J. Numer. Anal., 21 (2001), pp. 439-450.

[15] C. Huang and S. Vandewalle, Stability of Runge-Kutta-Pouzet Methods for Volterra Integral and Integro-differential Equations with Delays, Tech. Rep. TW 361, Department of Computer Science, Katholieke Universiteit Leuven, Leuven, Belgium, 2003.

[16] T. Кото, Stability of Runge-Kutta methods for delay integro-differential equations, J. Comput. Appl. Math., 145 (2002), pp. 483-492.

[17] S. MASET, Stability of Runge-Kutta methods for linear delay differential equations, Numer. Math., 87 (2000), pp. 355-371.

[18] P. J. van Der Houwen AND B. P. Sommeijer, Stability in linear multistep methods for pure delay equations, J. Comput. Appl. Math., 10 (1984), pp. 55-63.

[19] J. Wu, Theory and Applications of Partial Functional Differential Equations, Springer-Verlag, New York, 1996.

[20] B. Zubik-Kowal, Stability in the numerical solution of linear parabolic equations with a delay term, BIT, 41 (2001), pp. 191-206.

[21] B. Zubik-Kowal and S. VAndeWalle, Waveform relaxation for functional-differential equations, SIAM J. Sci. Comput., 21 (1999), pp. 207-226. 